\title{
Cervical fusion cage computationally optimized with porous architected Titanium for minimized subsidence
}

\author{
Ahmed Moussa $^{\mathrm{a}}$, Michael Tanzer ${ }^{\mathrm{b}}$, Damiano Pasini $^{\mathrm{a}}$ \\ ${ }^{a}$ Department of Mechanical Engineering, McGill University, Montreal, Quebec, Canada \\ H3AOC3 \\ ${ }^{b}$ Division of Orthopaedics, Department of Surgery, McGill University, Jo Miller Orthopaedic \\ Research Laboratory, Montreal, Quebec, H3G1A4, Canada
}

\begin{abstract}
Anterior cervical discectomy with fusion is a common surgical treatment that can relieve patients suffering from cervical spondylosis. This surgery is most commonly performed with the use of a cervical cage. One serious complication of the fusion cages commercially available in the market is subsidence of the cage with loss of the normal alignment of the spine and recurrent pain. This work presents the proof-of-concept of a fusion cage made of a graded porous titanium with microarchitecture minimizing the risk of subsidence associated with fully-solid implants. The optimized properties of the porous implant are obtained through a scheme combining multiscale mechanics and density-based topology optimization. Asymptotic homogenization is used to capture the effective properties of the porous material, which uses a tetrahedron based cell as building block. The stress levels and normal strains obtained under various loading conditions on the $\mathrm{C} 7$ superior surface of the vertebrae are used as indicators of subsidence. The results suggest a reduced risk of subsidence for the optimized implant versus the fully-solid implant. Under the most severe condition of combined loading, a collective improvement of the average von Mises stress up to $14 \%$ can be observed on the posterior, left, and right lateral regions of the $\mathrm{C} 7$ superior surface. Similarly, for the average normal strain, the optimized cage exhibits a more favourable distribution with a top gain of $21.7 \%$ at given locations.
\end{abstract}

Keywords: subsidence, fusion cage, homogenization, topology optimization, porous materials, lattice architecture, mechanical metamaterials. 


\section{Introduction}

Age-related degeneration of the cervical spine is the most common cause of neural disorder and has been reported that disc degeneration is common in over $50 \%$ of middle-aged individuals $[1$, 2]. Although most patients are asymptomatic, disc herniation, osteophyte formation and hypertrophied ligaments can compress the cervical spinal cord and nerve roots resulting in cervical pain, radiculopathy, or myelopathy [3]. If the physiotherapy or medications fail to relieve these symptoms, surgery is usually recommended. Among several surgical treatments, an anterior cervical discectomy with fusion (ACDF) is widely used to remove the herniated or degenerated disc in the neck [4]. ACDF has been shown clinically successful in more than $90 \%$ of patients, by alleviating their pain and allowing them to return to work [5]. Historically, ACDF was accomplished by removing the compressing structures [6] and wedging a bone block, harvested from the iliac crest of the patient (autograft), between the vertebral bodies. Although autologous bone graft is considered to be the gold standard in achieving fusion, the associated morbidity in harvesting the graft has motivated the search for alternative implants and materials. Since the fusion cage technology was proposed by Bagby in 1988, stand-alone cage designs, with or without additional fixation, have become the standard of ACDF. Cages avoid the morbidity associated with harvesting autogenous bone graft, and have been demonstrated success in achieving primary stability and long-term fusion $[1,7]$. Although excellent results have been reported with cages, subsidence of the cage has been reported as a complication in $3 \%$ to $10 \%$, of the cases [8]. Subsidence occurs when the implant protrudes through the adjacent vertebral body. Many reasons can lead to subsidence, such as inadequate determination of preoperative bone quality, and improper prosthesis design, which affects end plate preparation and load distribution [9].

Ideally, interbody cage implants should have materials with improved biomechanical properties, be biocompatible and promote osseointegration [10]. Different materials have been used to manufacture cervical cages, including three main materials: 1) Titanium (Ti) and its alloys, 2) Polyetheretherketone (PEEK), and 3) Carbon fiber-PEEK. Ti and PEEK are preferred in current designs, since synovitis and lymphatic spread of fiber debris has been associated with radiolucent carbon fiber-PEEK cages, although all three materials have their advantages and disadvantages [11-14]. Titanium and its alloys are biocompatible materials with high stiffness, corrosion resistance, and low density [15]. Ti cages have demonstrated their ability to support osseointegration [16]. However, the mismatch in elastic modulus of the Ti material with the adjacent bone results in stress shielding [17]. The PEEK cages can provide the advantages of radiolucency and lower subsidence rates than Ti cages [18]. Their elastic modulus is close to that of the bone, hence avoiding the stress shielding associated with Ti cages [17, 19-22]. However, PEEK cages without additional instrumentation or bone grafting, have been found to have a prolonged time to fusion and the fusion is usually incomplete [22, 23]. 
A number of studies using in vitro and numerical approaches have investigated the various reasons for cervical cage subsidence, with the modulus mismatch being one of the most prominent. Among the former group, Wilke et al. [24, 25] and Kettler et al. [26] have examined the role of neck movements on the subsidence of a set of commercially available cages, i.e. the WING cage (Medinorm AG, Quierschied, Germany), the BAK/C cage (Sulzer SpineTech, USA), and the AcroMed cervical I/F cage (DePuy AcroMed International, UK). Their results show the significant role of postoperative neck movements, with the WING and the AcroMed cages having lower subsidence than the BAK/C cage. Another in vitro study by Furderer et al. [27] on bovine vertebrae has compared subsidence caused by a selected number of cage designs under prescribed loading conditions. Abrasion of the vertebral endplates has been recognized as one cause for increased subsidence. Among the second group of investigations using numerical techniques, Lin et al. [28] have used a commercial software package to generate a lumbar interbody fusion cage with a porous architecture optimized for structural stability, reduced stress shielding, and biofactor delivery. Compared with conventional threaded cages, their design claims reduced stress shielding and lower stress at the cage-vertebra interface, thereby resulting in low subsidence. Another numerical study has compared the performance of selected cervical cages in the market, each with its own geometry and materials: Bryan (Medtronic Sofamor Danek, Minneapolis, MN), Prestige LP (Medtronic Sofamor Danek), and ProDisc-C (Synthes, Inc., West Chester, PA) [29]. The results contribute to better understand the underlying mechanisms causing cage subsidence, which is evaluated numerically through stress predictions at the vertebrae-prosthesis interface. Among the designs, the Bryan disc featuring a very complaint core with no cage fixators shows the lowest stresses superior to $\mathrm{C} 6$, which results in a reduced risk of subsidence. Chiang et al. [30] have also used finite element analysis to understand the role of loading, cage geometry and material, along with bone mineral density on the mechanism of subsidence of two prostheses: the SOLIS (Stryker Instruments, Kalamazoo, USA) and the BAK/C (Sulzer SpineTech, USA). The results show substantial subsidence under extension load, and suggest the cause for this problem to the mismatch of material properties between the cage and the adjacent cervical vertebrae.

This paper presents a proof-of-concept fusion cage that reduces the mismatch of elastic properties with the native bone, thereby reducing the risk of subsidence associated with fullysolid implants currently available in the market. Density-based topology optimization is used to tune the elastic properties of a porous microarchitected cage featuring tetrahedron based cell, here chosen for both its load bearing and bone ingrowth characteristics [31]. Asymptotic homogenization $[32,33]$ is used to capture the mechanical properties of the representative volume element (RVE), as a function of its relative density. The topology optimization problem is formulated for maximum implant compliance (strain energy) under a set of constraints, which include the overall porosity range of the cellular implant, bone ingrowth, stability, and additive manufacturing requirements. Five loading modes are considered as a combination of compression with either flexion, extension, right lateral bending, flexion combined with right lateral bending, or extension combined with right lateral bending moments, and the pertinent 
stress levels, as well as normal strains, are assessed on the $\mathrm{C} 7$ superior surface with values indicating the potential for subsidence reduction of the optimized porous cage versus its fullysolid counterpart. In section 2 , the article first presents the methodology to generate the computation model from the CT-scan data and the material properties assignment, and then describes the problem formulation for constrained topology optimization. In section 3 , the results, i.e. von Mises stress and normal strains distribution, are presented and a comparison of the optimized versus the fully-solid implant is given for the five load cases. A discussion follows in section 3 framing the results within the broad clinical context and highlighting current limitations and future work.

\section{Methodology}

One of the main cause of subsidence is the mismatch of elastic properties between the implant and surrounding native bone $[34,35]$. This work proposes to tune the elasticity gradients of the former to achieve mechanical biocompatibility with the latter while guaranteeing the satisfaction of strength requirements. Topology optimization is used for the purpose and applied to a threedimensional domain replicating the implant macrogeometry $\left(11 \times 14 \times 5 \mathrm{~mm}, 7^{\circ}\right.$ Lordotic angle $)$ of a commercially available cage (Trabecular Metal TM-S Cervical Fusion Device, Zimmer Spine, Minneapolis, MN, USA). Figure 1 briefly depicts the scheme here presented, where the key steps rely on combining concepts of multiscale mechanics and density-based topology optimization, as briefly summarized below:

- Acquisition of patient vertebral geometry and elastic tissue properties. CT-scan data of a 59-year-old female are obtained from the database "visible human project" (VHP) provided by the US national library of medicine (NLM, Bethesda, Maryland, USA).

- Reconstruction of functional spinal unit and assignment of material properties. After segmentation of the CT slices at the C6-C7 levels, the three-dimensional geometry of the C6-C7 vertebrae is reconstructed and assembled along with the implant and bone graft. This operation allows to create a complete functional spinal unit (FSU), where bone material properties are assigned based on the Hounsfield Unit values $(H U)$ of the voxels of the CT data (see Appendix B).

- Unit cell geometry and mechanical properties of porous Titanium. An open cell with tetrahedron based topology is chosen as building block of the cage porous architecture. The choice is motivated by the proven capabilities of this cell to provide both load bearing capability and bone ingrowth [31]. Asymptotic homogenization [32, 33, 36, 37] is used to calculate the homogenized stiffness tensor and yield properties (section 2.2. and Appendix D) of the unit cell, with characteristic length much smaller than the implant. The implant material properties are assigned to each tetrahedron unit cell as a function of its relative density. A uniform distribution of relative density is initially assigned. 
- Finite element model. A 3D finite element analysis (FEA) is used for the FSU under prescribed loads and boundary conditions that replicate the normal physiological range of the cervical vertebrae. Five loading cases are considered as shown in Figure 1(a), and for each of them the distribution of stress, strain, displacement, as well as strain energy is obtained over the whole spinal unit.

- Elastic properties tuning. To minimize implant subsidence into the vertebral endplate, the implant compliance, i.e. strain energy, is maximized (Figure 1(b)) via topology optimization. The design variable is the relative density, $\rho$, of each element of the cage, which is updated using the standard optimality criteria (OC) method [38]. A set of constraints is imposed on the overall porosity range of the cellular implant to account for bone ingrowth requirements and manufacturing constraints. The displacements at each node of a mesh element along with the homogenized properties of the unit cell are then retrieved to build the global stiffness tensor. The gradient of the objective function is then obtained through partially taking the gradient of the stiffness tensor with respect to its relative density (see Appendix A). The optimization process continues until convergence is reached, i.e. the attainment of the optimized relative density distribution that achieves the objective and satisfies the constraints (Appendix C). Failure analysis is also performed to satisfy strength requirements along with the calculation of the relevant safety factors (Appendix D).

We remark that the scheme here presented is fully automatic and integrated, with inhouse routines (MATLAB, MathWorks ${ }^{\circledR}$, Natick, Massachusetts, United States) developed to calculate the material properties of the vertebrae and the other components of the spinal unit, as well as to optimize and generate the fusion cage architectures. Computation time varies with the selected loading case. For example, for the fully-solid case, 40 minutes to 60 minutes are needed to obtain the solution, whereas for the optimized cages, the solution converges after 3 to 4 hours of iterations. 
(a) Finite element model

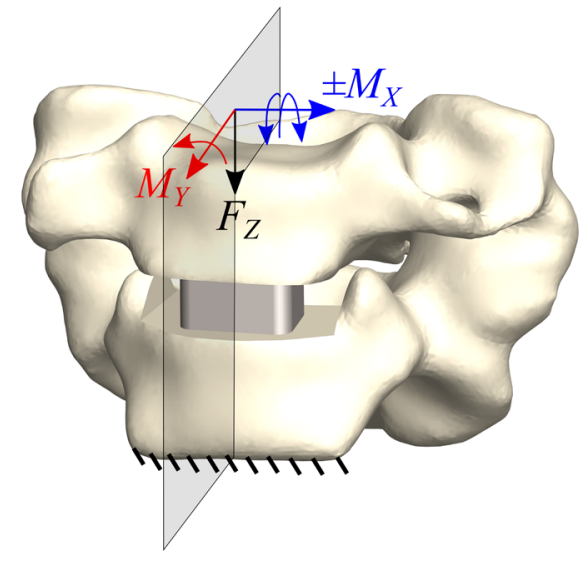

(b) Elastic properties tuning via topology optimization
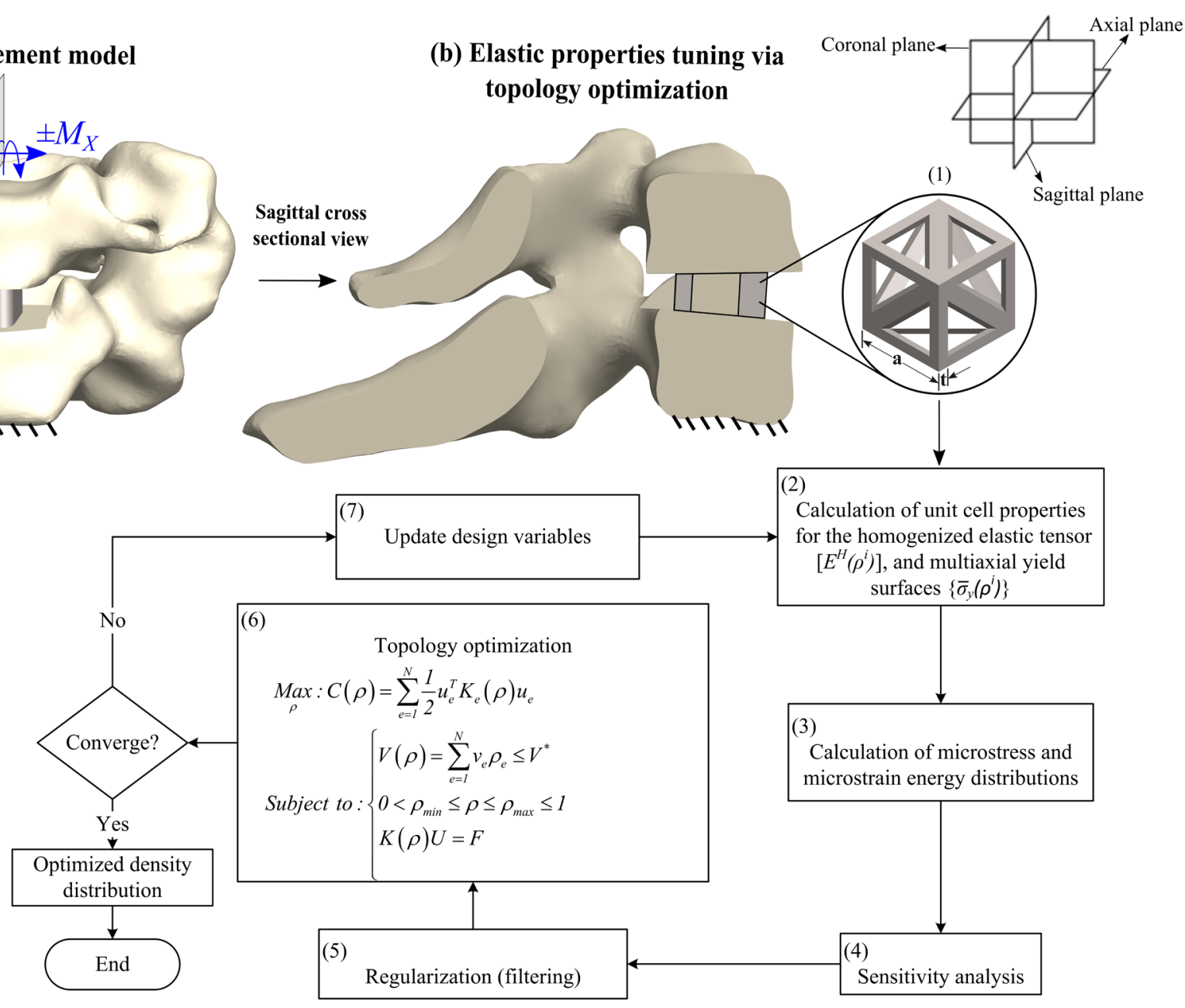

Figure 1: (a) FE model creation for a complete functional spinal unit after implant and bone graft insertion, with applied loads (compressive force $F_{Z}$, flexion/extension moments $\pm M_{X}$, and right lateral bending moment $M_{Y}$ ) and boundary conditions (fixed degrees of freedom of $\mathrm{C} 7$ inferior endplate); (b) Flow chart illustrating the analysis and design scheme used to develop the graded cellular fusion cage minimizing subsidence via topology optimization.

\subsection{Finite element model}

The geometry of the C6 and C7 vertebrae is reconstructed from the CT dataset of a 59-year-old female (NLM, Bethesda, Maryland, USA). The spacing and pixel size of the slices of interest (labeled from 1214 to 1257 ) are $1 \mathrm{~mm}$ and $0.8594 \mathrm{~mm}$ respectively. Imported into ITK-SNAP [39], the stack of CT images are used via a semi-automated segmentation process to create the vertebral geometry including the facet joints. Further, smoothing is performed on the volume mesh to reduce noise and sharp edges. A mesh file with STereoLithography (STL) format, a standard file format for solid freeform fabrication, is exported to MeshLab [40] to create a point cloud file for the vertebral model. The 3D geometry is then created from the point clouds by using the software package SolidWorks ${ }^{\circledR}$ (Dassault Systèmes SolidWorks Corporation). Threedimensional models of implant and bone graft are also created independently, and later carefully positioned to replicate the conditions of implant insertion during surgery (Figure 1(a)). The 
process of implant and bone graft location relies on firstly removing each vertebral endplate. Then, the implant and bone graft endplates are placed inferior to $\mathrm{C} 6$ and superior to $\mathrm{C} 7$, so that the cancellous bone is exposed and in contact with both the implant and bone graft endplates. Through Boolean operations between components, which have geometric overlap, a complete model is generated with geometry that mimics the way a surgeon deburrs the bone before implant insertion. The complete functional spinal unit (FSU) consisting of the C6-C7 vertebral bone, facet joints, implant, and bone graft, is then exported to Altair HyperWorks ${ }^{\circledR}$ software package (Troy, Michigan, United States) to create the volume mesh, with prescribed node sets at the locations of the applied loads and the domain boundary. The mesh consists of ten-node quadratic tetrahedron elements that can easily fill complex geometries, such as the spinal unit, without sacrificing the element quality [41]. The final model compromises 153054 elements and 239743 nodes is analyzed with the software package ANSYS $^{\circledR}$ (Canonsburg, Pennsylvania, U.S.A).

Table 1 shows the material properties of the C6-C7 vertebrae, as well as the other spinal unit components. The material properties of the cervical vertebrae are calculated under the assumption that the bone is transverse isotropic [34, 42, 43]; $E_{X}, E_{Y}$, and $E_{Z}$ are the Young's moduli in the $X, Y$, and $Z$ directions, while $v_{X Y}, v_{Y Z}$, and $v_{X Z}$ are the Poisson's ratios in the $X Y, Y Z$, and $X Z$ planes respectively. The axial elastic modulus $E_{Z}$ of each element is calculated through the relation between the elastic modulus and the bone mineral density (BMD) [34, 44]. The Hounsfield values $(H U)$ are measured from the CT images and a linear interpolation between $H U$ and BMD is assumed to determine BMD (Appendix B). Bone graft and facet joints are assumed to have isotropic material properties, while Titanium alloy [28] (Ti6Al4V) is the base material that is used to build the fusion cage. The bone graft is assumed load bearing, and a perfectly bonded contact between implant, bone graft, and C6-C7 vertebrae is modelled to represent the complete fusion of the cage with the vertebrae.

Table 1: Material properties of the functional spinal unit components

\begin{tabular}{|l|c|c|c|c|}
\hline $\begin{array}{l}\text { Material property/ } \\
\text { Component }\end{array}$ & $\begin{array}{c}\text { Bone properties of C6-C7 vertebrae } \\
{[34,42-44]}\end{array}$ & $\begin{array}{c}\text { Bone graft } \\
\text { cancellous bone } \\
\text { properties [28] }\end{array}$ & $\begin{array}{c}\text { Tissue } \\
\text { properties of } \\
\text { facet joints [29] }\end{array}$ & $\begin{array}{c}\text { Implant } \\
\text { Titanium alloy } \\
\text { (Ti6Al4V [28] }\end{array}$ \\
\hline $\begin{array}{l}\text { Young's Modulus, } \\
E(M P a)\end{array}$ & $\begin{array}{l}E_{Z}=-34.7+3230 B M D_{Q C T} \\
E_{X}=E_{Y}=0.333 E_{Z}\end{array}$ & 100 & 5 & 120000 \\
\hline $\begin{array}{l}\text { Poisson's Ratio, } v \\
v_{X Y}=0.381 ; v_{Y Z}=v_{X Z}=0.104\end{array}$ & 0.2 & 0.45 & 0.3 \\
\hline $\begin{array}{l}\text { Shear modulus, } G \\
(M P a)\end{array}$ & $\begin{array}{l}G_{X Y}=E_{X} / 2\left(1+v_{X Y}\right)=0.121 E_{Z} \\
G_{Y Z}=G_{X Z}=0.157 E_{Z}\end{array}$ & $\begin{array}{c}\text { The value of shear modulus is calculated from the } \\
\text { assumption that the material is isotropic. }\end{array}$ \\
\hline
\end{tabular}


Five systems of loads representing the normal physiological range (Sagittal flexion/extension moments, $M_{X}= \pm 1.8 \mathrm{Nm}$; right lateral bending moment, $M_{Y}=1.8 \mathrm{Nm}$, flexion combined with right lateral bending, and extension combined with right lateral bending moments, each with an axial compression, $F_{Z}=73.6 \mathrm{~N}$ ) are imposed on the superior of the C6 vertebra (Figure 1(a)) [45]. The compressive load denotes the equivalent weight of the head and neck. All the degrees of freedom at the inferior endplate of $\mathrm{C} 7$ are fixed. A master node is created to distribute the axial compressive force and moments across the nodes of the C6 superior endplate.

\subsection{Effective mechanical properties of the prosthesis}

A fully-detailed simulation of a porous domain, such as the cage here examined, requires the explicit modeling of all its microstructural features, a process that can be expensive and timeconsuming [37]. Here instead, we opt to treat the porous cage as a homogenized medium, whose effective properties are obtained from the unit cell, i.e. the Representative Volume Element (RVE). Asymptotic Homogenization (AH) $[32,33]$ is used for this purpose as described below.

\subsubsection{RVE mechanical properties}

The basic assumption of AH theory is that each field quantity depends on two different scales: one is the macroscopic (global) level $x$, and the other is the microscopic (local) level, $y=x / \eta . \eta$ is a magnification factor that scales the dimensions of the unit cell to the dimensions of the material at the macroscale. It also assumes that field quantities, such as displacement, stress, and strain, vary smoothly at the macroscopic level, and are periodic at the microscale [32, 33]. Based on $\mathrm{AH}$, each physical field, such as the displacement field, $u_{i}^{\eta}$, in a porous elastic body, can be expanded into a power series with respect to $\eta$ :

$$
u_{i}^{\eta}\left(x_{i}, y_{i}\right)=u_{0 i}\left(x_{i}, y_{i}\right)+\eta u_{1 i}\left(x_{i}, y_{i}\right)+\eta^{2} u_{2 i}\left(x_{i}, y_{i}\right)+\ldots \quad ; y_{i}=\frac{x_{i}}{\eta}
$$

where $u_{i}^{\eta}$ is the exact value of the field variable and $u_{0 i}$ is the macroscopic (average) value of the field variable. $u_{1 i}, u_{2 i}$ are perturbations in the field variables due to the periodic arrangement of the microstructure unit cells, $x_{i}$ are the global level coordinates, and $y_{i}$ are the micro level coordinates. $\mathrm{AH}$ is here used to calculate the elastic stiffness tensor as well as the strength properties for failure analysis. The former is explained below and the latter in Appendix D. The effective stress tensor $\bar{\sigma}_{i j}$ of the unit cell as a function of the average strain tensor $\bar{\varepsilon}_{k l}$ can be expressed through the effective elastic tensor $E_{i j k l}^{H}$ as:

$$
\bar{\sigma}_{i j}=E_{i j k l}^{H} \bar{\varepsilon}_{k l},
$$

Considering only the first order terms in equation (1), the effective stiffness tensor of the building block $E_{i j k l}^{H}$ can be obtained by solving a local problem formulated on the RVE and results in: 
$E_{i j k l}^{H}=\frac{1}{|Y|} \int_{Y_{s}} E_{i j p m} M_{p m k l} d Y$

where $|Y|$ is the total volume of the unit cell (including voids), $Y_{s}$ is the solid part of the cell, and $E_{i j p m}$ is the local elasticity tensor, which equals the elasticity tensor of the material in the solid domain of the cell and equals to zero for the void domain. In addition, the local structure tensor $M_{i j k l}$ relates the macroscopic average strain $\bar{\varepsilon}_{k l}$ to the microscopic strain $\varepsilon_{i j}$ as:

$\varepsilon_{i j}=M_{i j k l} \bar{\varepsilon}_{k l}, M_{i j k l}=\frac{1}{2}\left(\delta_{i k} \delta_{j l}+\delta_{i l} \delta_{j k}\right)-\varepsilon_{i j}^{* k l}$

where $\delta_{i j}$ is the Kronecker delta, and $\varepsilon_{i j}^{* k l}$ is the microscopic strain corresponding to the component $k l$ of the macroscopic stain tensor $\bar{\varepsilon}_{k l}$. Under the assumption of small deformation and linear elasticity, the microstructural strain $\varepsilon_{i j}^{* k l}$ is obtained by solving a local problem defined on the $\operatorname{RVE}[33,46]$ :

$\int_{Y_{s}} E_{i j p m} \varepsilon_{i j}^{1}(v) \varepsilon_{p m}^{* k l}(u) d Y=\int_{Y_{s}} E_{i j k l} \varepsilon_{i j}^{1}(v) \bar{\varepsilon}_{k l} d Y$

where $\varepsilon_{i j}^{1}(v)$ is the virtual strain. In three-dimensions, six arbitrary unit strains are required to construct the local structure tensor $M_{i j k l}$. Knowing that the periodicity of the strain field quantities at the microscale is ensured by applying periodic boundary conditions on the edges of the RVE, the nodal displacements on the opposite edges are equal $[33,47]$. Once the local structure tensor, $M_{i j k l}$, is obtained, the homogenized stiffness tensor, $E_{i j k l}^{H}$, of the lattice unite cell is calculated by substituting the structure tensor into (3) [33, 48, 49].

The procedure described above is applied to obtain the effective elastic properties of the tetrahedron based cell topology [49]. Figure 2 shows its effective elastic moduli normalized with the bulk properties of the solid, i.e. $E=120 \mathrm{GPa}$ and $v=0.3$, and expressed as a function of relative density $\rho$. Because of cubic symmetry, the elastic tensor of the tetrahedron based unit cell has three independent elastic constants [50]: Young's modulus $\left(E_{x x}\right)$, shear modulus $\left(G_{x y}\right)$, and Poisson's ratio $\left(v_{x y}\right)$. Appendix D provides details on the calculation of its effective yield properties along with the failure analysis. 


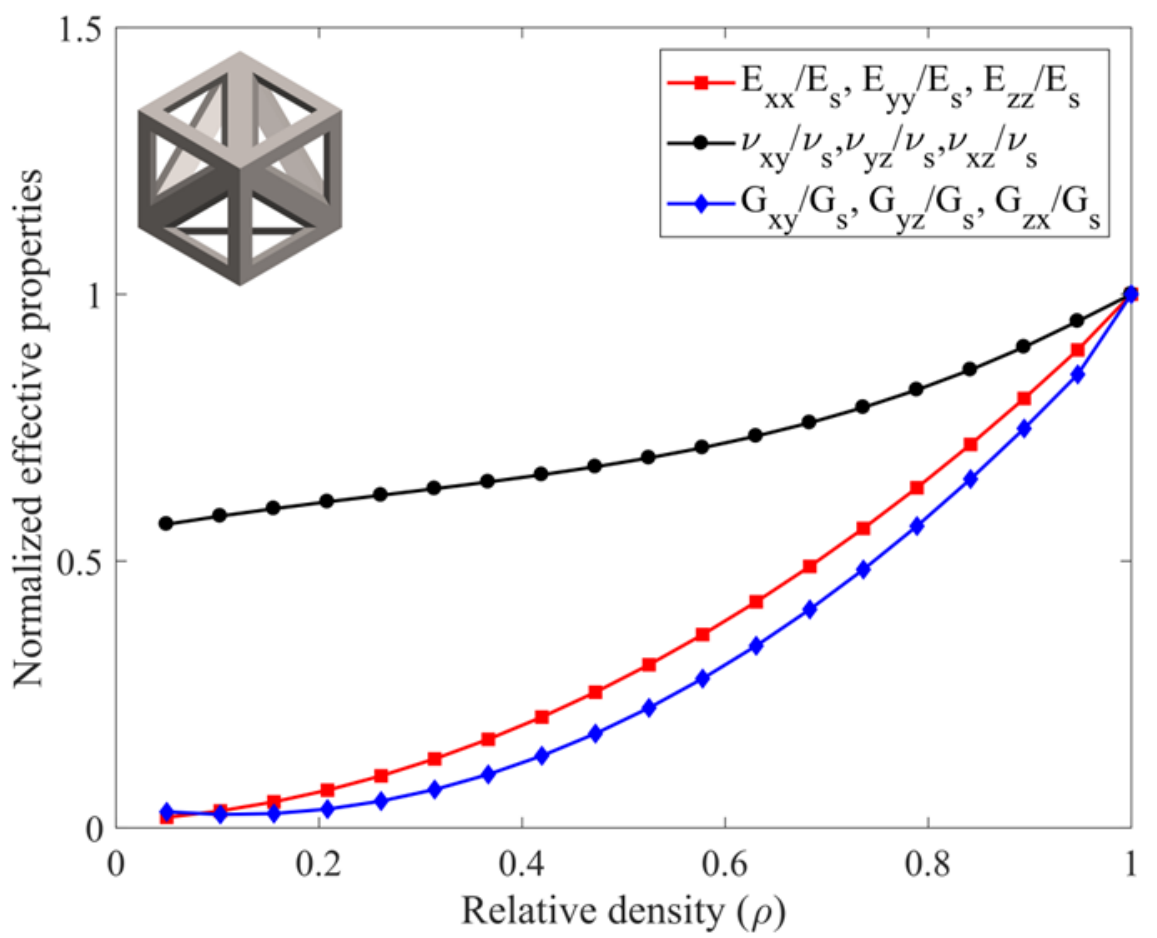

Figure 2: Normalized effective properties of the tetrahedron based unit cell as a function of relative density.

\subsection{Topology optimization}

A density-based topology optimization is used to find the optimized material distribution within the fusion cage subjected to the boundary conditions and external forces illustrated in Figure 1(a) [51]. The implant domain is cellular with unit cells having effective properties that can vary with relative density (Figure 2). Section 2.3.1 reports the problem formulation and the sensitivity analysis, while section 2.3.2 describes the design constraints that account for bone ingrowth and ensure manufacturability.

\subsubsection{Problem formulation and sensitivity analysis}

To avoid implant perforation in the cervical vertebra, we need to reduce subsidence to the minimum. This clinical goal can translate into the objective of minimizing the implant subsidence, which is generally caused by the mismatch in elastic properties between the native trabecular bone $[34,35]$ and the implant. Gradient tuning of implant elasticity, therefore, is one of the most effective means to eradicate the root of the problem; it contributes to lower the stress level at the bone-implant interface and reduce subsidence. In fact, if the implant elasticity is tailored to better match that of the native bone, lower levels of stress and strain would appear at the contact regions between the implant and the cancellous bone of the vertebrae. With a lower contact stress, as well as contact strain, at the bone-implant interface comes a reduced propensity of subsidence. In addition, the higher the compliance, or the strain energy, the lower the stiffness of the structure, a strategy equivalent to minimizing the mismatch of elastic moduli between the implant and the adjacent vertebrae. As such, to minimize subsidence, we aim at minimizing the 
implant stiffness, which is equivalent to maximize compliance, here expressed in terms of the applied loads and the state variables as:

$C(\rho)=\frac{1}{2} F^{T} U(\rho)$

where $F$ is the global force vector applied to the superior endplate of the C6 vertebra, and $U(\rho)$ is the global vector of nodal displacements, which represents the state variables. The global force vector can be expressed by the following state equation:

$F=K(\rho) U(\rho)$

where $K$ is the global stiffness matrix of the implant, which is the assembly of the elemental stiffness matrix of the implant microstructures, as described in Appendix A. The mathematical formulation of the problem is expressed as:

$$
\begin{gathered}
\underset{\rho}{\operatorname{Max}}: C(\rho)=\frac{1}{2} F^{T} U(\rho)=\sum_{e=1}^{N} \frac{1}{2} u_{e}^{T} K_{e}(\rho) u_{e} \\
\text { Subject to }:\left\{\begin{array}{l}
V(\rho)=\sum_{e=1}^{N} v_{e} \rho_{e} \leq V^{*} \\
0<\rho_{\min } \leq \rho \leq \rho_{\max } \leq 1 \\
K(\rho) U=F
\end{array}\right.
\end{gathered}
$$

wherein, $\rho$ is the relative density vector, $\rho_{e}$ is the relative density of each element, $V^{*}$ is the prescribed volume fraction of solid material, and $v_{e}$ is the volume of each element $e$, while $N$ is the total number of elements. The optimization problem in the standard minimization form is expressed as:

$$
\begin{aligned}
& \underset{\rho}{\text { Min }: C(\rho)=}-\frac{1}{2} F^{T} U(\rho)=-\sum_{e=1}^{N} \frac{1}{2} u_{e}^{T} K_{e}(\rho) u_{e} \\
& \text { Subject to }:\left\{\begin{array}{l}
V(\rho)=\sum_{e=1}^{N} v_{e} \rho_{e} \leq V^{*} \\
0<\rho_{\min } \leq \rho \leq \rho_{\max } \leq 1 \\
K(\rho) U=F
\end{array}\right.
\end{aligned}
$$

The standard optimality criteria (OC) method is used to solve the problem [38], after performing a sensitivity analysis that guides the search direction to the optimized solution. At the macroscopic level, the mean compliance depends on the material properties of each element, which are a function of the relative density of each element, $\rho_{e}$. Thus, the derivatives of the mean compliance with respect to the variation of $\rho_{e}$ in the element $e$ can be expressed using (6) and (7) as: 
$\frac{\partial C(\rho)}{\partial \rho_{e}}=-\frac{1}{2} F^{T} \frac{\partial U(\rho)}{\partial \rho_{e}}=-\frac{1}{2} U^{T}(\rho) K(\rho) \frac{\partial U(\rho)}{\partial \rho_{e}}$

Taking the derivatives of the state equation (7) with respect to the element densities $\rho_{e}$, knowing that $F$ has a constant value, yields:

$\frac{\partial K(\rho)}{\partial \rho_{e}} U(\rho)+K(\rho) \frac{\partial U(\rho)}{\partial \rho_{e}}=0$

where, $\partial U(\rho) / \partial \rho_{e}$ can be expressed as:

$\frac{\partial U(\rho)}{\partial \rho_{e}}=-K^{-1}(\rho) \frac{\partial K(\rho)}{\partial \rho_{e}} U(\rho)$

Substituting (12) into (10) yields to the final form of the sensitivity of the objective function with respect to the relative density $\rho_{e}$ :

$\frac{\partial C(\rho)}{\partial \rho_{e}}=\frac{1}{2} U^{T}(\rho) \frac{\partial K(\rho)}{\partial \rho_{e}} U(\rho)$

The derivatives of the stiffness matrix of the implant microstructure, described here by the tetrahedron unit cell, with respect to the design variables can be obtained by the scheme given in Appendix A. Once the stiffness matrix derivatives are calculated for each element, the global stiffness derivatives can be assembled and used to calculate the sensitivity vector of the objective function through equation (13). The sensitivity of the material volume $V$ with respect to the element density $\rho_{e}$ is expressed as:

$\frac{\partial V}{\partial \rho_{e}}=v_{s}$

where $v_{s}$ is the volume of the solid element.

To avoid numerical instabilities, such as checkerboard patterns and mesh dependency [52], we resort to a filtering method which can also serve to ensure manufacturability. Among several filtering techniques [53], in this paper we implement a mesh-independent sensitivity filtering [52, 54, 55]. To implement such a filter, the finite element problem is solved in the standard way. After calculating the sensitivities consistently, these are heuristically modified as weighted averages of the sensitivities in mesh-independent neighborhoods. Generally, the neighborhood of element $e\left(N_{e}\right)$ is specified by the elements that have centers within a given filter radius $R$ of the center of element $e$ :

$N_{e}=\left\{i \mid\left\|x_{i}-x_{e}\right\| \leq R\right\}$

where, $x_{i}$ and $x_{e}$ are the central coordinates of elements $i$ and $e$ respectively. The sensitivity filter modifies the sensitivities $\partial C(\rho) / \partial \rho_{e}$ as follows: 


$$
\frac{\widehat{\partial C(\rho)}}{\partial \rho_{e}}=\frac{\sum_{i \in N_{e}} w\left(x_{i}\right) \rho_{i} \frac{\partial C(\rho)}{\partial \rho_{i}}}{\rho_{e} \sum_{i \in N_{e}} w\left(x_{i}\right)}
$$

wherein, $\rho_{e}$ and $\rho_{i}$ are the density of element $e$ and its neighbouring element $i$ respectively; and $w\left(x_{i}\right)$ is a weighting function that is given by the linearly decaying function:

$$
w\left(x_{i}\right)=R-\left\|x_{i}-x_{e}\right\|
$$

Once the sensitivity of the objective function is obtained and filtering is applied, the design variables are updated using the optimality criteria (OC) method until convergence is achieved. Failure analysis then follows in the process as per the details given in Appendix D.

\subsubsection{Definition of bone ingrowth and manufacturing constraints}

Bone ingrowth requirements and manufacturing restrictions should be satisfied to guarantee the clinical functionality and manufacturability of the fusion cage via additive manufacturing. These can be set as constraints on the design variable, the relative density $\rho$. As recommended clinically, the relative density of the top and bottom surfaces of the porous implant (Figure 3(b) is set to $30 \%$, a value that promotes bone ingrowth, which should initiate after surgery at the upper and lower surfaces of the cages in contact with the inferior and superior sides of C6 and C7, respectively [31, 49]. Furthermore, the relative density of the interior surface of the implant, surrounding the bone graft, is set above $50 \%$ so as to ensure proper stability and, concurrently, favor osteogenesis and osteoconduction around the graft.

As per the manufacturing constraints, recent studies on additive manufacturing of porous bone replacement implants [31, 49], have identified the interplay between manufacturability and bone ingrowth, a relation that can be specified by constraints on the unit cell geometry. In particular for the tetrahedron based unit cell, the minimum strut thickness should be above $200 \mu \mathrm{m}$ and the maximum pore size less than $800 \mu \mathrm{m}$. Figure 3(c) shows that for a constant cell size, $a$, of 1.2 $\mathrm{mm}$, a value of relative density between 0.25 to 0.8 can be obtained with a uniform strut thickness and pore size. The strut thickness and pore size variations are between 200-441 $\mu \mathrm{m}$ and 267- $503 \mu \mathrm{m}$ respectively, ranges that satisfy manufacturing and bone ingrowth constraints as demonstrated in previous studies [31, 56, 57]. Figure 3(d) shows the relevant effective properties (section 2.2.1) that correspond to the constrained range of relative density. These constraints along with prescribed volume fraction $V^{*}$ of $50 \%$ for the solid material and a filter radius of 1.5 times the element size, are factored in to solve the problem until convergence is reached under the five loading modes that combine compression with either flexion, extension, right lateral bending, flexion combined with right lateral bending, or extension combined with right lateral bending moments (Appendix C). As described in the following section, an optimized distribution of relative density is obtained for the load cases, each achieving the objective, satisfying the constraints and ensuring the necessary strength requirements. 
(a)

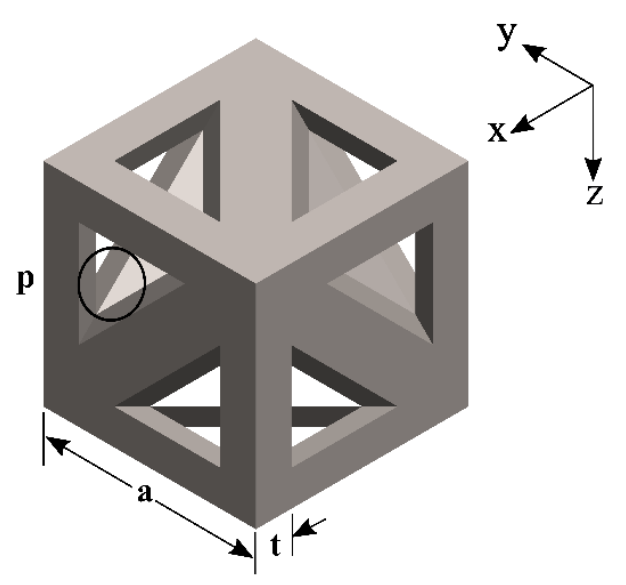

(c)

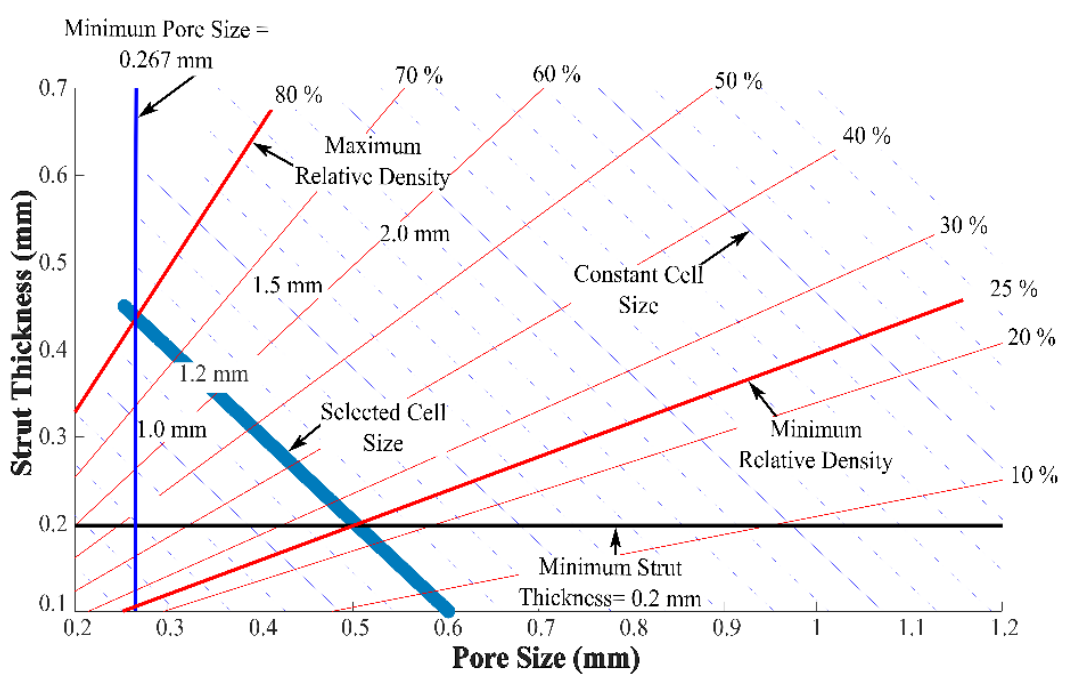

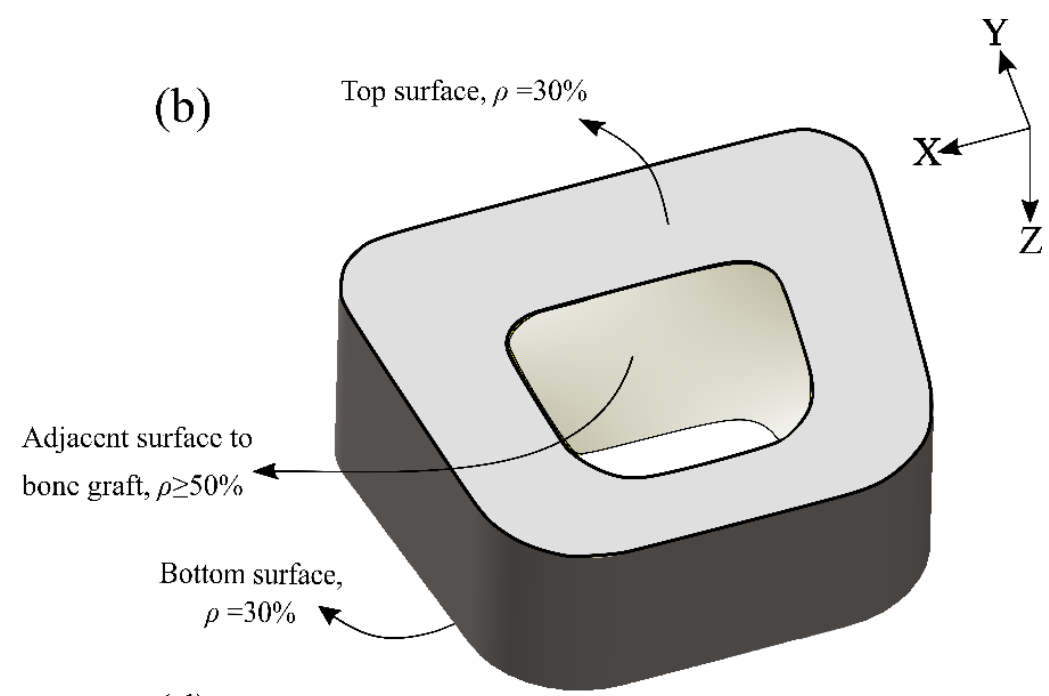

(d)

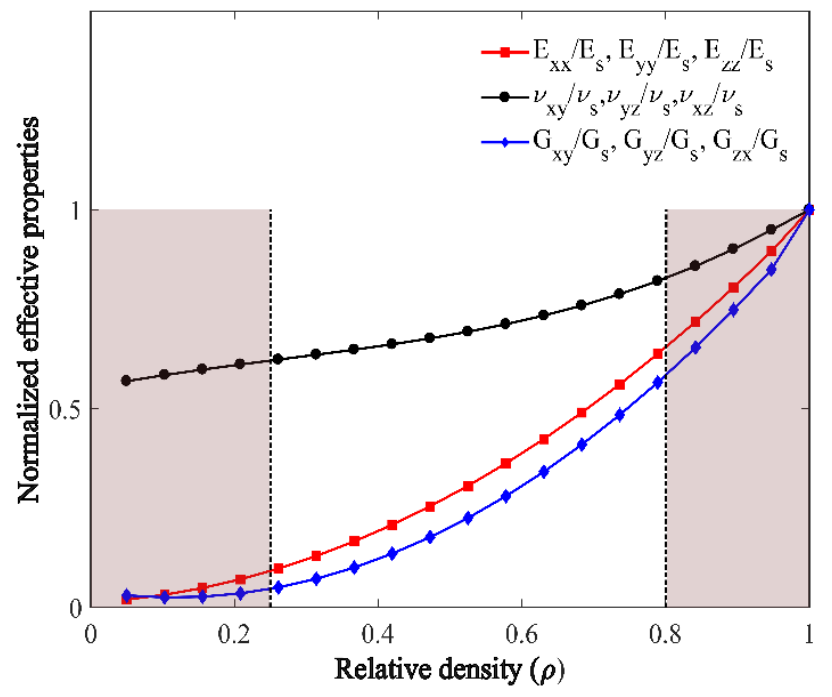

Figure 3: Identification of bone ingrowth and additive manufacturing constraints of the porous cage. (a) Tetrahedron based unit cell; (b) Bone ingrowth and stability requirements; (c) Additive manufacturing constraints for the selected cell size, a, of $1.2 \mathrm{~mm}$; (d) Effective elastic moduli corresponding to the constrained range of relative density. 


\section{Results and discussion}

Figure 4 ((a-e) top) shows the optimized relative density distribution obtained under the $1.8 \mathrm{Nm}$ flexion, extension, right lateral bending, flexion combined with right lateral bending, and extension combined with right lateral bending moments, each accompanied by a compressive force of $73.6 \mathrm{~N}$. The bottom rows illustrate their respective porous cage that results by mapping the optimized relative density into a lattice microarchitecture built from a tetrahedron based unit. The implants are obtained through in-house python scripts developed for the purpose (Rhinoceros, McNeel North America, Seattle, WA).

(a)
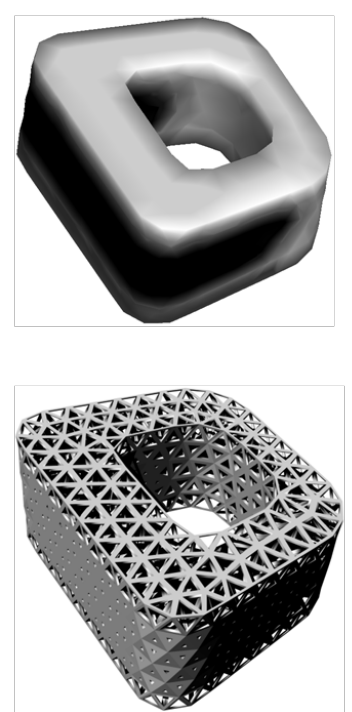

(d)
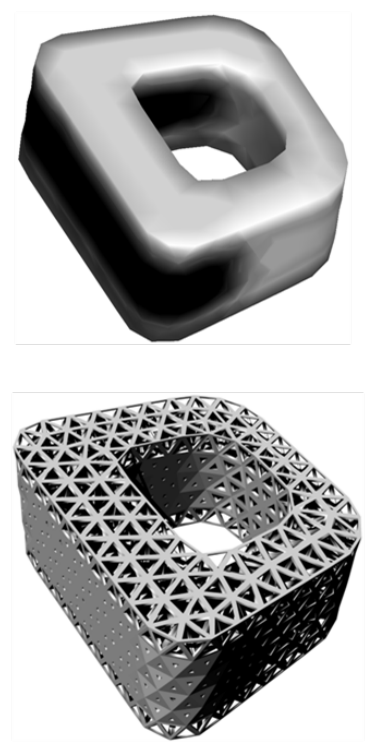

(b)
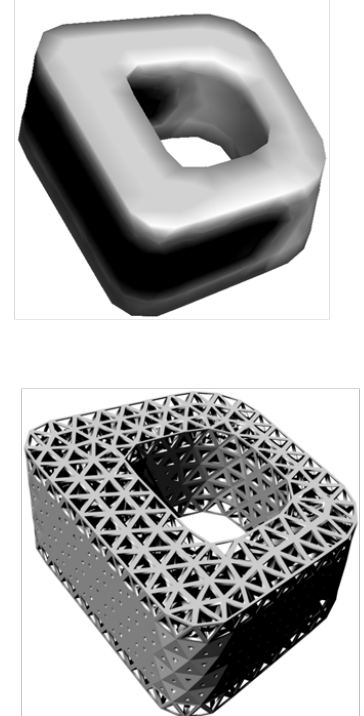

(e)
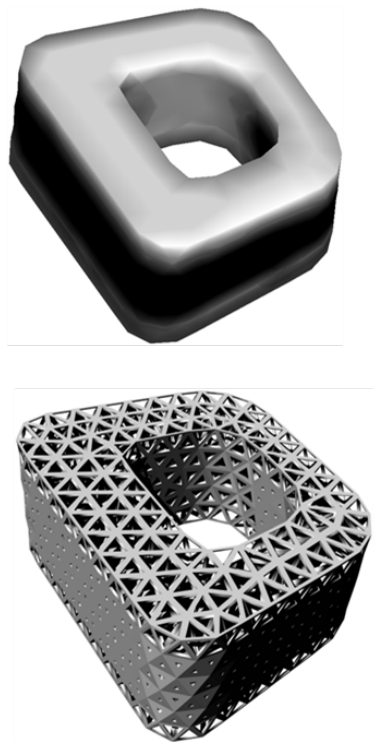

(c)
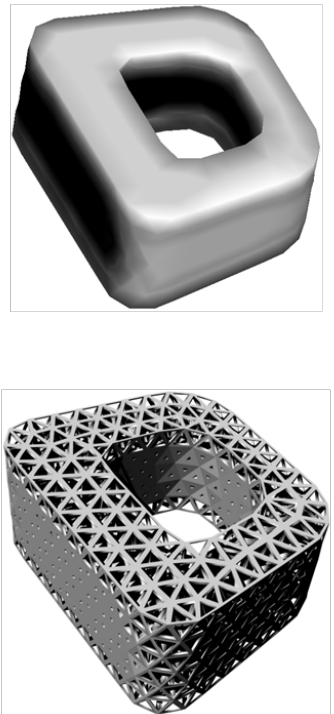

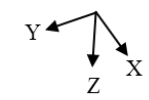

Figure 4: Optimized relative density distribution (top) and its corresponding lattice architecture (bottom) for (a) Flexion, (b) Extension, (c) Right lateral bending, (d) Flexion combined with right lateral bending, and (e) Extension combined with right lateral bending modes. 
To assess implant subsidence, in this work we use the von Mises stress and normal strains on the C7 superior surface. While there is no direct measure of subsidence, von Mises stress has been proved to correlate with the onset of subsidence [29, 30]. Based on the assumption that a high stress concentration results in a higher propensity of subsidence in the long-term [29], the von Mises stress levels are used as a proxy for subsidence. In particular, the fully-solid fusion cage is here taken as baseline, and its von Mises stress is compared to that of the optimized cage on the C7 superior surface. The strains, in the normal direction of the C7 superior surface, are also considered as another metric that contributes to evaluate any propensity.

Overall, the von Mises stress level over the C7 superior surface is shown much higher in the model with the fully-solid implant than the optimized porous implant. Figure 5 shows the von Mises stress distributions on the superior surface of the $\mathrm{C} 7$ vertebra under flexion, extension, right lateral bending, flexion combined with right lateral bending, and extension combined with right lateral bending load cases, with a compressive force of $73.6 \mathrm{~N}$ representing the weight of the head and the neck. A symmetric distribution of von Mises stress is observed for the first three load cases, whereas an asymmetric pattern emerges for the combined loadings. Noticeably, the location of the maximum von Mises stress is dependent on the loading case. Following the standard anatomical terms of location, the maximum von Mises stress is located in the anterior region of the case of flexion (yellow) and flexion combined with right lateral bending (red); while in case of extension, the maximum von Mises stress is in the posterior region (red). In the case of right lateral bending and extension combined with right lateral bending modes, the maximum value is located in the right lateral region (red areas).

Qualitative measures of the stress distribution on the C7 superior surface show that in the posterior region the porous cage can produce lower stress concentration than the solid one, for both flexion, extension, and flexion combined with right lateral bending load cases, and in the left lateral region in the case of lateral bending. In the case of extension combined with right lateral bending load case, it appears in the right lateral region. On the other hand, no significant difference appears for the von Mises stress distributions in the other regions. In terms of difference in the average von Mises stress distribution over the posterior, left, and right lateral regions, outlined by dashed boxes in Figure 5, the optimized implant shows $11 \%, 11.8 \%, 13.7 \%$, $12.6 \%$, and $14 \%$ lower stress than the fully-solid implant, in flexion, extension, right lateral bending, flexion combined with right lateral bending, and extension combined with right lateral bending respectively. Moreover, numerical results of the optimized implant show that the effective Young's modulus of the elements that are in contact with the C7 superior surface are reduced from $120 \mathrm{GPa}$, the Young's modulus of the bulk material, to about $14 \mathrm{GPa}$. This can be easily observed in Figure 2 and Figure 3(d) at relative density $\rho=30 \%$. The reduced mismatch of elastic properties between the porous implant microstructure and the native bone $[34,35]$ results in low stress concentration compared to the fully-solid implant. Hence, the tendency of the porous implant to subside into the $\mathrm{C} 7$ superior surface becomes lower. 
A study by Goel et al. [58] thoroughly described the loads transmitted through the facet joints and intervertebral disc in the case of flexion, extension, and lateral bending modes, with compressive force. The predicted stress distributions from the work undertaken here agree with their conclusions: for a flexion moment of $1.8 \mathrm{Nm}$ in conjunction with a compressive force of $73.6 \mathrm{~N}, 113 \%$ of the load is carried by the disc, while the facet joints are unloaded. On the other hand, each of the facets carries $51 \%$ of the loading, whereas the disc receives only $14 \%$, in the case of extension with compression load. This explains the reason for the lower values of von Mises stress found over the $\mathrm{C} 7$ superior surface for extension compared to the flexion load case. For the right lateral bending mode, the right facet withstands $41 \%$ of the loading, whereas the left facet is unloaded, and the disc receives only $68 \%$. This corroborates the findings that the maximum von Mises stress levels are located in the right lateral region of the $\mathrm{C} 7$ superior surface of the vertebral bone. To conclude, the predicted von Mises stress is higher in the combination of flexion and axial compression, compared to the extension and right lateral bending load cases. Overall, the highest predicted von Mises stress occurs in the case of flexion combined with right lateral bending accompanied by axial compression. 

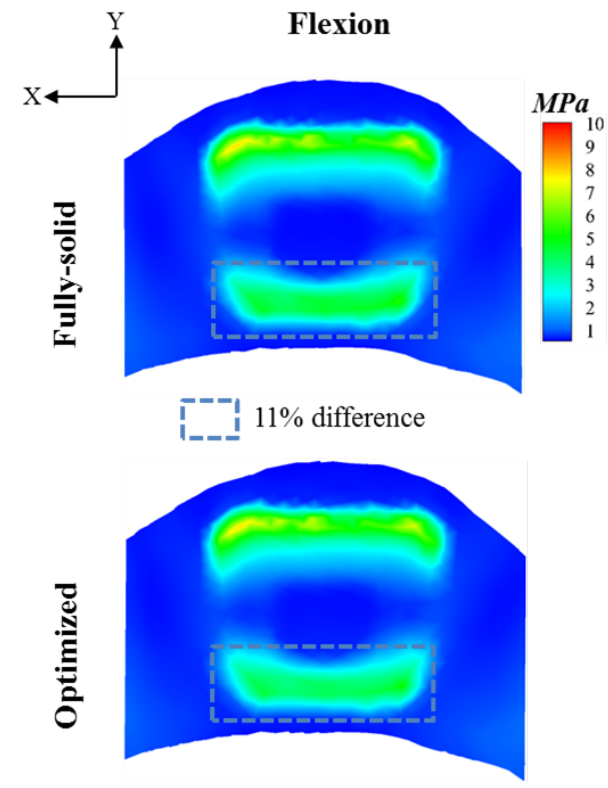

Flexion + Right lateral bending

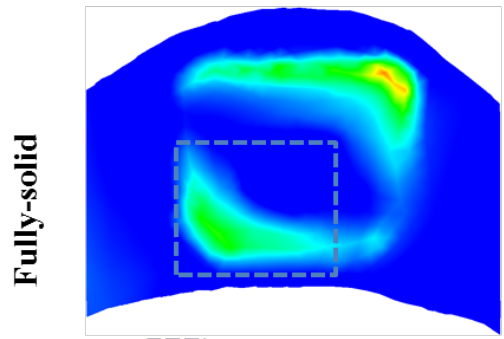

$\mid 12.6 \%$ difference

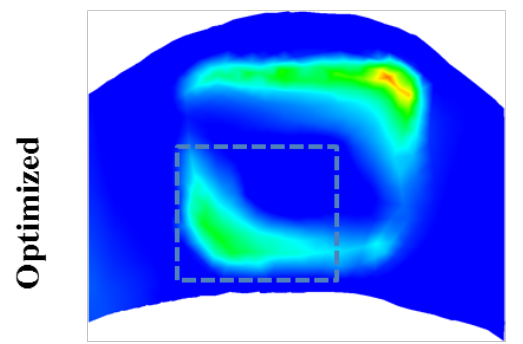

Extension

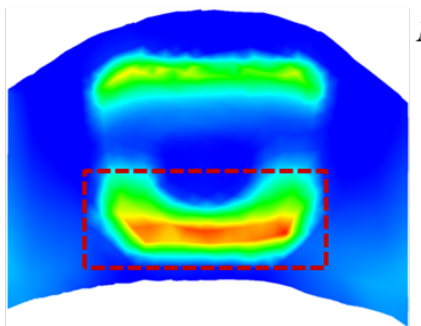

$11.8 \%$ difference

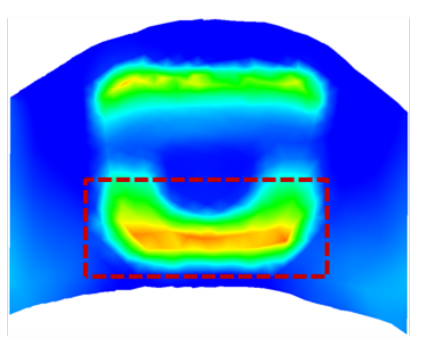

Lateral bending
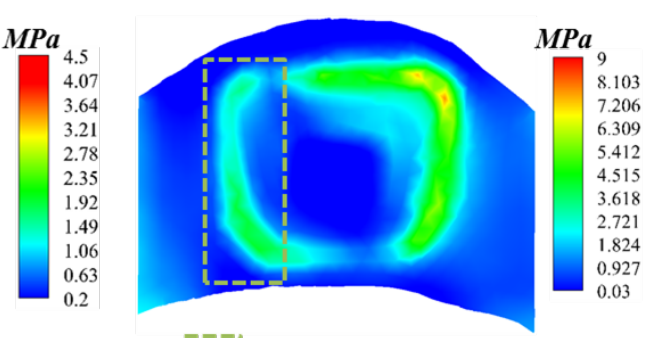

$13.7 \%$ difference

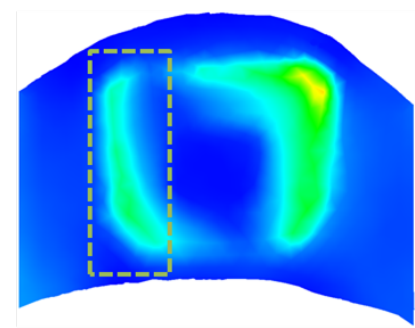

Extension + Right lateral bending
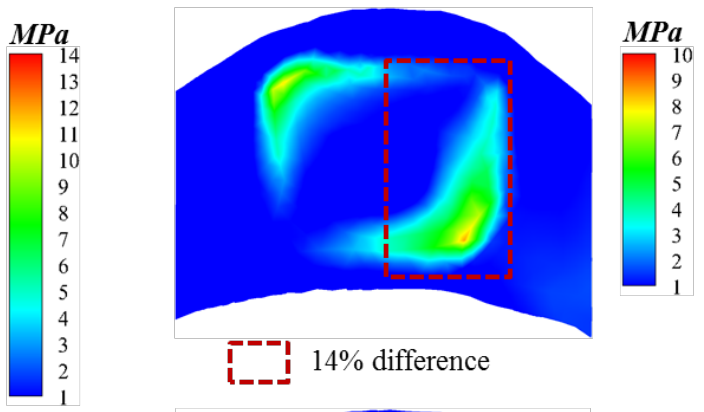

\section{I_- $14 \%$ difference}

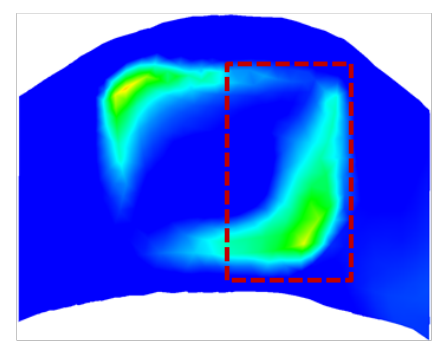

Figure 5: Comparison of von Mises stresses (MPa) on cancellous bone of the C7 superior surface, using fullysolid and optimized prostheses in flexion, extension, right lateral bending, flexion combined with right lateral bending, and extension combined with right lateral bending modes.

To further evaluate the tendency of subsidence, the normal strain on the superior surface of the C7 vertebra is used here as another metric. Figure 6 shows the strain distributions for the fullysolid and optimized prostheses, under the load cases examined. Similarly, the distribution of normal strain is symmetric for the first three load cases, as opposed to the combined load cases. In the five loading modes, similar to Figure 5, a lower strain is observed, overall, using the optimized porous implant compared to the fully-solid. By comparing the three separate load 
cases of flexion, extension and right lateral bending, the highest predicted strains on the superior surface of the $\mathrm{C} 7$ vertebra appear when the spinal unit is placed under axial compression and flexion. By comparing all five loading modes, the highest predicted strains on the superior surface of the $\mathrm{C} 7$ vertebra appear when the spinal unit is placed under axial compression and flexion combined with right lateral bending moment. In a previous study [58], the maximum intradisc pressure was found in the flexion $(1.8 \mathrm{Nm})$ with axial compression $(73.6 \mathrm{~N})$ load case, which agrees with the findings of this work for identical conditions of loading. This might be clinically explained by looking at the behavior of the intact vertebral model of the human being. In the case of flexion moment and compressive force, the intervertebral disc is compressed, while the posterior ligaments, such as interspinous and capsular ligaments, are under tension. On the other hand, ligaments are slackened in the extension load case. This means that, for the flexion moment with axial compression, the highest strains are in the interspinous ligament [58]. In terms of difference in the average normal strain distribution over the posterior, left, and right lateral regions, outlined by the dashed boxes in Figure 6, the optimized implant shows 18.5\%, $20 \%, 11 \%, 19.4 \%$, and $21.7 \%$ lower strain than the fully-solid implant in Z-direction, in flexion, extension, right lateral bending, flexion combined with right lateral bending, and extension combined with right lateral bending respectively. This agrees with the conclusion gained from evaluating the risk of subsidence using the von Mises stress as a metric: a higher stress, or strain, concentration on the bone surface results in higher tendency of the implant to subside into the adjacent surface of the vertebral bone.

The five concepts introduced in this work for a porous cage with minimized levels of stress and strain at the vertebrae surfaces are obtained through implant elasticity tuning that guarantees the strength sufficient to carry the physiological set of loads the cage is subjected to. To avoid implant failure, the Tsai-Wu failure criterion is used to calculate the safety factor, and among the optimized concepts, the weakest is 3.25 , a value that corresponds to the load case of flexion combined with right lateral bending moment (Appendix D). 

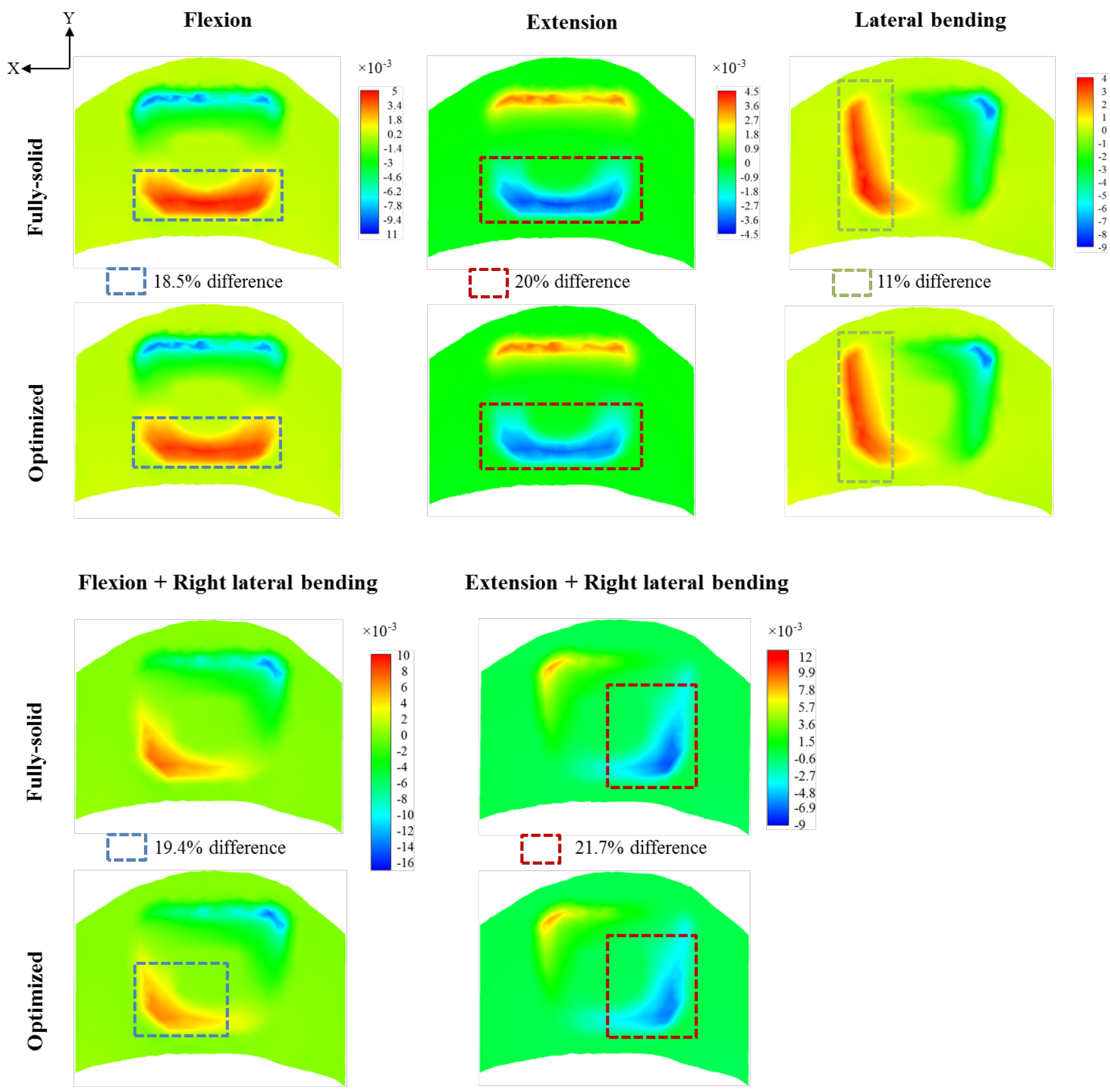

\section{Extension + Right lateral bending}
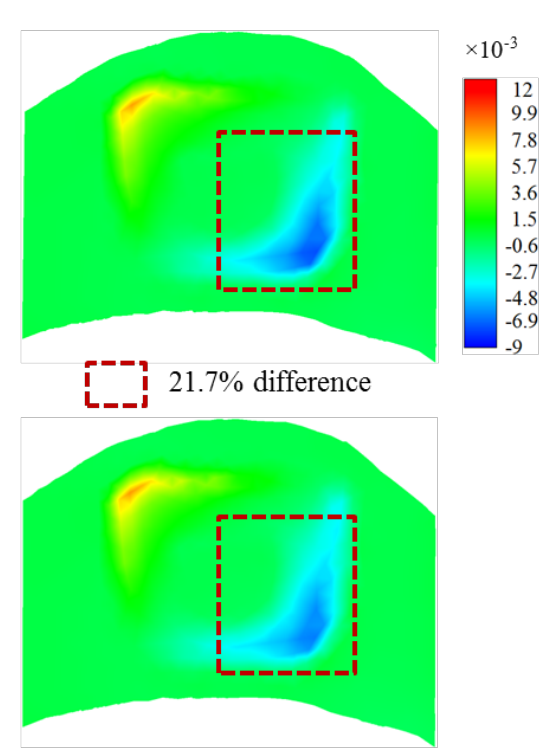

Figure 6: Comparison of the normal strain on cancellous bone of the $\mathrm{C} 7$ superior surface, using fully-solid and optimized prostheses in flexion, extension, right lateral bending, flexion combined with right lateral bending, and extension combined with right lateral bending modes.

The numeric study here undertaken bears some limitations that need to be addressed in future work. Since the porous cage is treated as a homogenized medium, the approach here presented to calculate the effective properties does not account for the localized stress and strain induced by the interaction between cage struts adjacent vertebrae. In addition, the assumptions dealing with an unbounded domain is not reflected by the finite size of the implant, a factor not captured by asymptotic homogenization theory which assumes an infinitely periodic domain with no 
boundary effects. To address this issue, a multiscale finite element method is proposed to more accurately capture the mechanical properties of the porous implant [59]. A fully-detailed FEA of the porous implant could also be used to assess the accuracy of the homogenized models here presented. Moreover, the results presented in this numeric study should be validated by experiments on additively manufactured implants.

Another point pertains to uncertainties, which can arise from a number of sources including unknown origin of the patient bone, variation of trabeculae properties, patient gender and age, location in the body, mineral content, disease, e.g. osteoporosis, as well as imperfections from manufacturing. In this work, we consider variability in the material properties of the cervical vertebrae. From a real CT-scan data of a 59-year-old female, the material properties are calculated as a function of the bone mineral density (BMD) [34, 44], under the assumption of transverse isotropy for bone tissue [34, 42, 43]. Appendix B provides the pertinent details. Imperfections emerging from additive manufacturing play also an important role as recently demonstrated $[31,56,57]$ but are not here considered. On the front of fabrication, cellular implants with tailored gradients of porosity and pore morphology that enhance bone ingrowth and tune performance have been recently demonstrated [31, 49, 60-65]. For example, a fully porous hip implant featuring a lattice microstructure similar to the one presented in this paper has been recently manufactured through SLM and successfully tested in vitro [31, 49]. Its graded microarchitecture features a minimum strut thickness of $200 \mu \mathrm{m}$ and a maximum pore size of $800 \mu \mathrm{m}$, characteristics that are built through AM, which are similar to the constraints assigned to the fusion cage implant presented in section 2.3.2 [31, 49]. These previous works, therefore, suggests the feasibility of additively manufacturing the cage implant reported in this study. 


\section{Conclusions}

This paper has presented a numeric investigation aiming at improving the performance of commercially available fusion cages made of solid materials. It proposes to reduce their risk of subsidence by adopting a porous material architecture with properties tuned to smooth out the mismatch in elastic properties between the implant and the adjacent vertebrae. For the purpose, topology optimization has been formulated to achieve maximum implant compliance. The numerical results suggest a decrease of interface stresses and strains. The location of both maximum von Mises stress and normal strain has been observed to be dependent on the loading case, in accordance with the intact model of the human. The highest von Mises stress and normal strain, on the superior surface of the $\mathrm{C} 7$ vertebra, is predicted to occur when the spinal unit is subjected to compression and flexion combined with right lateral bending moment, compared to the other load cases. The average von Mises stress distribution over the posterior, left, and right lateral regions on the $\mathrm{C} 7$ superior surface is about $11 \%, 11.8 \%, 13.7 \%, 12.6 \%$, and $14 \%$ lower for the optimized implant versus the fully-solid implant, under compressive force of $73.6 \mathrm{~N}$, in conjunction with $1.8 \mathrm{Nm}$ flexion, extension, right lateral bending, flexion combined with right lateral bending, and extension combined with right lateral bending moments respectively. Similarly, the average normal strain distribution over the posterior, left, and right lateral regions on the C7 superior surface is approximately $18.5 \%, 20 \%, 11 \%, 19.4 \%$, and $21.7 \%$ lower for the optimized implant versus the fully-solid implant, under the same loading conditions. The numeric results here presented suggest optimal porosity tuning can be effective in reducing the probability of subsidence into the superior surface of the $\mathrm{C} 7$ vertebral bone in comparison to the fully-solid implant. The numeric results presented in this paper warrants further experimental and clinical validation, work currently undertaken. 


\section{Appendix A: Calculation of stiffness tensor sensitivity of the implant microstructure}

To compute the sensitivity of the implant compliance (Section 2.3.1), the derivatives of the stiffness tensor need to be computed for the entire implant microstructure. This appendix presents a detailed derivation of the sensitivity of the stiffness tensor of the implant microstructure with respect to the design variables, the relative density of each element $\rho$. A tennode isoparametric quadratic tetrahedral element [66] (Table A.1) is used to discretize the finite domain of the implant described in Figure 1, as it can be easily adapted to complex geometries such as the vertebral bone.

Table A.1: Ten-node isoparametric quadratic tetrahedral element with node numbering conventions and Gauss points, in the rst natural coordinate system, $w_{k}$ is the weight of the Gauss point $k(k=1 \ldots, 5)$.

\begin{tabular}{|c|c|c|c|c|c|c|c|c|}
\hline Node & $r$ & $S$ & $t$ & $\begin{array}{c}\text { Gauss point } \\
(k)\end{array}$ & $\begin{array}{c}\text { Weight } \\
\left(w_{k}\right)\end{array}$ & $r_{k}$ & $s_{k}$ & $\boldsymbol{t}_{k}$ \\
\hline 1 & 0 & 1 & 0 & \multirow[t]{2}{*}{ 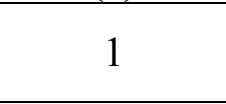 } & \multirow{2}{*}{$-2 / 15$} & \multirow{2}{*}{$1 / 4$} & \multirow{2}{*}{$1 / 4$} & \multirow{2}{*}{$1 / 4$} \\
\hline 2 & 0 & 0 & 1 & & & & & \\
\hline 3 & 1 & 0 & 0 & \multirow{2}{*}{2} & \multirow{2}{*}{$3 / 40$} & \multirow{2}{*}{$1 / 6$} & \multirow{2}{*}{$1 / 6$} & \multirow{2}{*}{$1 / 6$} \\
\hline 4 & 0 & 0 & 0 & & & & & \\
\hline 5 & 0 & 0.5 & 0.5 & \multirow{2}{*}{3} & \multirow{2}{*}{$3 / 40$} & \multirow{2}{*}{$1 / 6$} & \multirow{2}{*}{$1 / 6$} & \multirow{2}{*}{$1 / 2$} \\
\hline 6 & 0.5 & 0 & 0.5 & & & & & \\
\hline 7 & 0.5 & 0.5 & 0 & \multirow{2}{*}{4} & \multirow{2}{*}{$3 / 40$} & \multirow{2}{*}{$1 / 6$} & \multirow{2}{*}{$1 / 2$} & \multirow{2}{*}{$1 / 6$} \\
\hline 8 & 0 & 0.5 & 0 & & & & & \\
\hline 9 & 0 & 0 & 0.5 & \multirow{2}{*}{5} & \multirow{2}{*}{$3 / 40$} & \multirow{2}{*}{$1 / 2$} & \multirow{2}{*}{$1 / 6$} & \multirow{2}{*}{$1 / 6$} \\
\hline 10 & 0.5 & 0 & 0 & & & & & \\
\hline
\end{tabular}

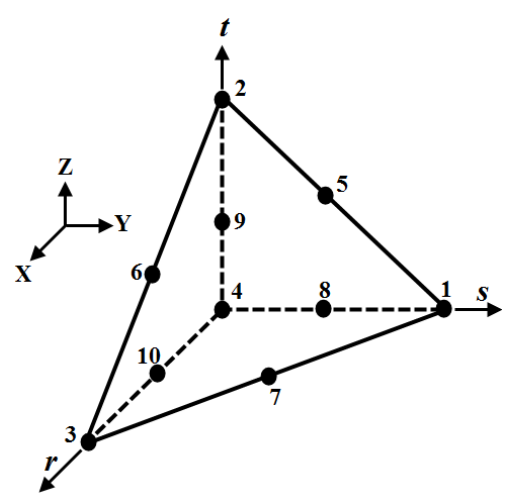

The shape functions of each node can be represented by the general quadratic polynomial of nodal displacements as:

$u_{i}(r, s, t)=a r^{2}+b s^{2}+c t^{2}+d r s+e s t+f r t+g r+h s+i t+J$

where $a, b, c, \ldots J$ are the constants of the polynomial, which can be determined by solving equation A.1 at each node $i(i=1 \ldots, 10)$ of the tetrahedron. Then, the shape functions of the tetrahedron element with respect to the natural coordinate system given by $r, s$, and $t$ can be obtained as: 


$$
\begin{aligned}
& N_{1}=2 s^{2}-s \\
& N_{2}=2 t^{2}-t \\
& N_{3}=2 r^{2}-r \\
& N_{4}=2 r^{2}+4 r s+4 r t-3 r+2 s^{2}+4 s t-3 s+2 t^{2}-3 t+1 \\
& N_{5}=4 s t \\
& N_{6}=4 r t \\
& N_{7}=4 r s \\
& N_{8}=4 s-4 r s-4 s t-4 s^{2} \\
& N_{9}=4 t-4 r t-4 s t-4 t^{2} \\
& N_{10}=4 r-4 r s-4 r t-4 r^{2}
\end{aligned}
$$

The direct stiffness approach is used to find the global stiffness tensor $K$, where the implant is discretized into small elements and the elemental stiffness matrix $K_{e}$ of each element is calculated before the global stiffness matrix, $K$, assembly, where $K_{e}$ is expressed as:

$$
K_{e}(\rho)=\int_{\Omega^{e}} B^{T} E^{H}(\rho) B d \Omega^{e}=\iiint_{x y z} B^{T} E^{H}(\rho) B d x d y d z
$$

where $B$ is the strain-displacement matrix and $E^{H}$ is the homogenized elastic tensor of each element $e$ (see section 2.2.1), and $\Omega^{e}$ is the volume of the element in the global coordinates. Since the strain-displacement matrix is independent of the design variables, the derivatives of the elemental stiffness matrix with respect to the design variables (relative density of each element $\left.\rho_{e}\right)$ can be expressed as follows:

$\frac{\partial K_{e}(\rho)}{\partial \rho_{e}}=\int_{\Omega^{e}} B^{T} \frac{\partial E^{H}(\rho)}{\partial \rho_{e}} B d \Omega^{e}=\iiint_{x y z} B^{T} \frac{\partial E^{H}(\rho)}{\partial \rho_{e}} B d x d y d z$

Generally, each mesh element corresponds to a tetrahedron-based cell that has three planes of symmetry; therefore 9 elastic constants are needed in the constitutive equations: 3 Young's moduli $E_{x x}, E_{y y}, E_{z z}, 3$ Poisson's ratios $v_{y z}, v_{z x}, v_{x y}$ and 3 shear moduli $G_{y z}, G_{z x}, G_{x y}$. The elastic stiffness tensor of the unit cell can thus be expressed as:

$$
\begin{aligned}
& E^{H}(\rho)=\left[\begin{array}{lllllll}
\frac{1-v_{y z}(\rho) v_{z y}(\rho)}{E_{y y}(\rho) E_{z z}(\rho) \Delta} & \frac{v_{y x}(\rho)+v_{z x}(\rho) v_{y z}(\rho)}{E_{y y}(\rho) E_{z z}(\rho) \Delta(\rho)} & \frac{v_{z x}(\rho)+v_{y x}(\rho) v_{z y}(\rho)}{E_{y y}(\rho) E_{z z}(\rho) \Delta(\rho)} & 0 & 0 & 0 \\
\frac{v_{x y}(\rho)+v_{x z}(\rho) v_{z y}(\rho)}{E_{z z}(\rho) E_{x x}(\rho) \Delta(\rho)} & \frac{1-v_{z x}(\rho) v_{x z}(\rho)}{E_{z z}(\rho) E_{x x}(\rho) \Delta(\rho)} & \frac{v_{z y}(\rho)-v_{z x}(\rho) v_{x y}(\rho)}{E_{z z}(\rho) E_{x x}(\rho) \Delta(\rho)} & 0 & 0 & 0 \\
\frac{v_{x z}(\rho)+v_{x y}(\rho) v_{y z}(\rho)}{E_{x x}(\rho) E_{y y}(\rho) \Delta(\rho)} & \frac{v_{y z}(\rho)+v_{x z}(\rho) v_{y x}(\rho)}{E_{x x}(\rho) E_{y y}(\rho) \Delta(\rho)} & \frac{1-v_{x y}(\rho) v_{y x}(\rho)}{E_{x x}(\rho) E_{y y}(\rho) \Delta(\rho)} & 0 & 0 & 0 \\
0 & 0 & 0 & 2 G_{y z}(\rho) & 0 & 0 & \\
0 & 0 & 0 & 2 G_{z x}(\rho) & 0 & & \\
0 & 0 & 0 & 0 & 2 G_{y x}(\rho) \\
\Delta(\rho)=\frac{\left[-v_{x y}(\rho) v_{y x}(\rho)-v_{y z}(\rho) v_{z y}(\rho)-v_{z x}(\rho) v_{x z}(\rho)-2 v_{y z}(\rho) v_{z x}(\rho) v_{x y}(\rho)\right.}{E_{x x}(\rho) E_{y y}(\rho) E_{z z}(\rho)}
\end{array}\right.
\end{aligned}
$$


Since asymptotic homogenization method is used to calculate the elastic constants of the unit cell across a range of relative densities (Figure 3(d)), we can represent the elastic tensor of each element as a function of the element relative density and then use it to evaluate the derivative of the elastic tensor with respect to the design variables. The strain-displacement matrix $B$ is also derived by using the shape function partials with respect to displacements in the global coordinate system. The strain-displacement matrix can be written as:

$B=\left[\begin{array}{llllllllll}B_{I} & B_{2} & B_{3} & B_{4} & B_{5} & B_{6} & B_{7} & B_{8} & B_{9} & B_{10}\end{array}\right]$

with:

$B_{i}=\left[\begin{array}{lll}N_{i, x} & 0 & 0 \\ 0 & N_{i, y} & 0 \\ 0 & 0 & N_{i, z} \\ N_{i, y} & N_{i, x} & 0 \\ 0 & N_{i, z} & N_{i, y} \\ N_{i, z} & 0 & N_{i, x}\end{array}\right], i=1, \ldots, 10$

where $N_{i, x}, N_{i, y}$ and $N_{i, z}$ are the derivative of the shape functions with respect to the global coordinate system. To construct the strain-displacement matrix, the derivative of the shape functions with respect to natural coordinate system, given by $r, s$, and $t$, is computed via the chain rule as:

$$
\begin{aligned}
& \frac{\partial N_{i}}{\partial r}=\frac{\partial N_{i}}{\partial x} \frac{\partial x}{\partial r}+\frac{\partial N_{i}}{\partial y} \frac{\partial y}{\partial r}+\frac{\partial N_{i}}{\partial z} \frac{\partial z}{\partial r} \\
& \frac{\partial N_{i}}{\partial s}=\frac{\partial N_{i}}{\partial x} \frac{\partial x}{\partial s}+\frac{\partial N_{i}}{\partial y} \frac{\partial y}{\partial s}+\frac{\partial N_{i}}{\partial z} \frac{\partial z}{\partial s} \\
& \frac{\partial N_{i}}{\partial t}=\frac{\partial N_{i}}{\partial x} \frac{\partial x}{\partial t}+\frac{\partial N_{i}}{\partial y} \frac{\partial y}{\partial t}+\frac{\partial N_{i}}{\partial z} \frac{\partial z}{\partial t}
\end{aligned}
$$

Then, arranging equations A. 8 in matrix form results in the derivative of the shape functions with respect to the global coordinate system:

$$
\left[\begin{array}{c}
\frac{\partial N_{i}}{\partial x} \\
\frac{\partial N_{i}}{\partial y} \\
\frac{\partial N_{i}}{\partial z}
\end{array}\right]=[J]^{-1}\left[\begin{array}{c}
\frac{\partial N_{i}}{\partial r} \\
\frac{\partial N_{i}}{\partial s} \\
\frac{\partial N_{i}}{\partial t}
\end{array}\right], \quad[J]=\left[\begin{array}{lll}
\frac{\partial x}{\partial r} & \frac{\partial y}{\partial r} & \frac{\partial z}{\partial r} \\
\frac{\partial x}{\partial s} & \frac{\partial y}{\partial s} & \frac{\partial z}{\partial s} \\
\frac{\partial x}{\partial t} & \frac{\partial y}{\partial t} & \frac{\partial z}{\partial t}
\end{array}\right]
$$

where $[J]$ is the Jacobian matrix. From (A.9), the Jacobian matrix of a ten-node Tetrahedron element may be expressed as: 


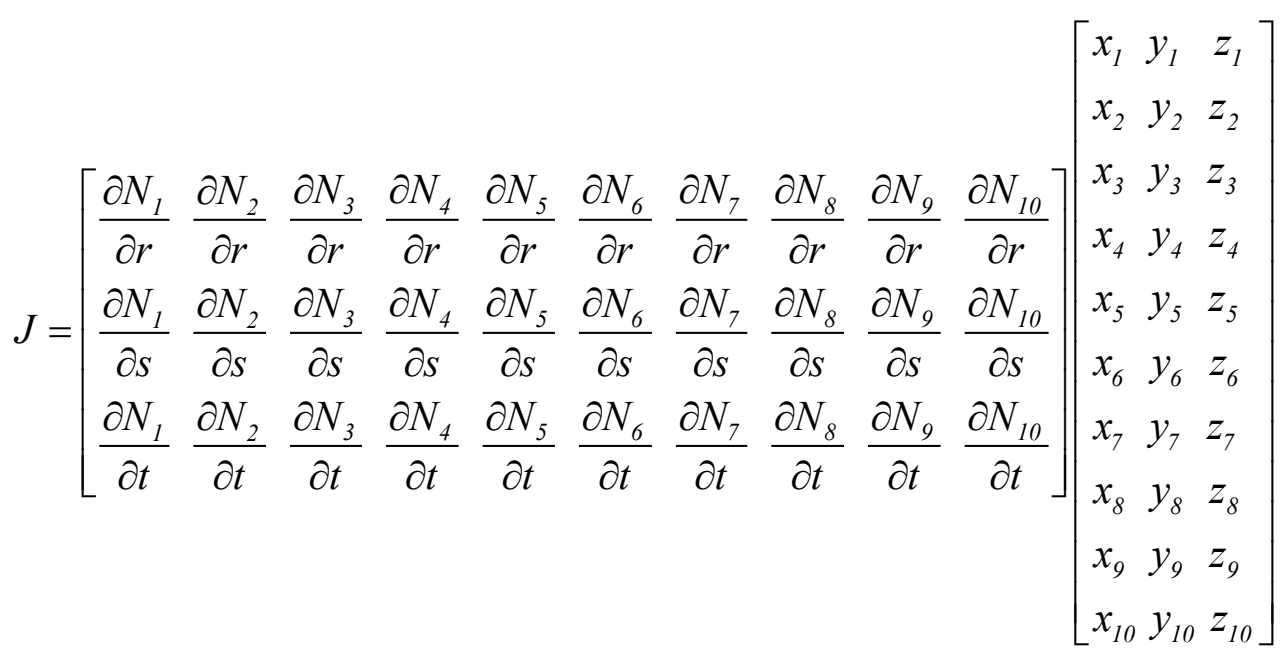

where $\left\{\left(x_{i}, y_{i}, z_{i}\right) \mid i \in(1,2, \ldots, 10)\right\}$ are the global coordinates of the element nodes. By substituting (A.9) into (A.7), we obtain the strain-displacement matrix for each element, from which the gradient of the stiffness matrix for the corresponding element is computed. For a ten-node tetrahedral element with five Gauss points (Table 1), and using the Gauss quadrature rule for volume integration, the elemental stiffness tensor equation A.3 and its derivatives A.4 can be rewritten as follows:

$$
\begin{aligned}
& K_{e}(\rho)=\int_{\Omega^{e}} B^{T} E^{H}(\rho) B d \Omega=\iiint_{x y z} B^{T} E^{H}(\rho) B d x d y d z \\
& =\iiint_{r s t} B_{r s t}^{T}(r, s, t) E^{H}(\rho) B_{r s t}(r, s, t)|J| d r d s d t=\frac{1}{6} \sum_{k=1}^{5} w_{k} B_{r s t}^{T}\left(r_{k}, s_{k}, t_{k}\right) E^{H}(\rho) B_{r s t}\left(r_{k}, s_{k}, t_{k}\right)|J| \\
& \frac{\partial K_{e}(\rho)}{\partial \rho_{e}}=\int_{\Omega^{e}} B^{T} \frac{\partial E^{H}(\rho)}{\partial \rho_{e}} B d \Omega=\iiint_{x y z} B^{T} \frac{\partial E^{H}(\rho)}{\partial \rho_{e}} B d x d y d z \\
& =\iiint_{r s t} B_{r s t}^{T}(r, s, t) \frac{\partial E^{H}(\rho)}{\partial \rho_{e}} B_{r s t}(r, s, t)|J| d r d s d t=\frac{1}{6} \sum_{k=1}^{5} w_{k} B_{r s t}^{T}\left(r_{k}, s_{k}, t_{k}\right) \frac{\partial E^{H}(\rho)}{\partial \rho_{e}} B_{r s t}\left(r_{k}, s_{k}, t_{k}\right)|J|
\end{aligned}
$$

where $k$ is the number of Gauss integration points in the parent tetrahedron. Once the stiffness matrix derivatives are calculated for each element using A.12, the global stiffness derivatives $\left(\partial K(\rho) / \partial \rho_{e}\right)$ can be assembled and used to calculate the compliance sensitivity vector $\left(\partial C(\rho) / \partial \rho_{e}\right)$ for the whole microarchitecture of the implant (see equation 13). The sensitivity analysis is then implemented under the optimization scheme described in section 2.3 .1 and shown in Figure 1(b), to seek the optimum relative density distribution of the cage. 


\section{Appendix B: Material property assignment from CT data}

After semi-automatic segmentation of the CT slices of the C6-C7 levels, the geometrical model is three-dimensionally reconstructed and assembled with the implant and bone graft. Figure B.1 shows the steps followed, starting from segmentation to a complete functional spinal unit (FSU) assembly. The finite element model of the cervical vertebral bone does not only rely on the accurate geometry, but also on the proper assignment of the bone material properties. From the high contrast between bone and soft tissue in the CT-data, we can obtain the relevant information [67-70]. The elastic properties of bone are calculated from the relationship between CT numbers, i.e. Hounsfield Unit $(H U)$ value of each voxel, and bone mineral density (BMD) [35, 67]. It has been noted that the mineral density of bone tissues has an approximate linear relationship with the $H U[34,35]$. In this work, the BMD does not account for both hydration and the organic phase of the bone.

(a) Segmentation

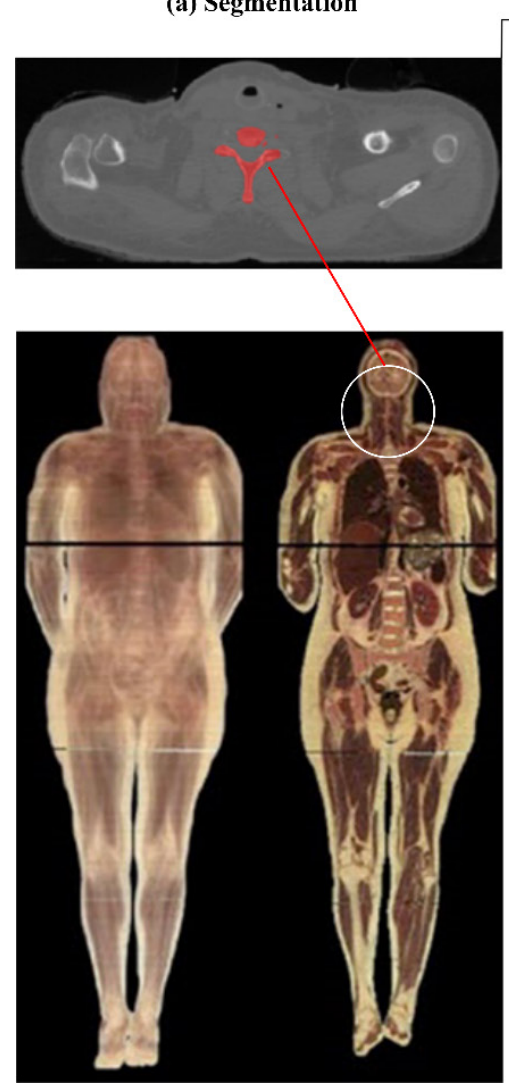

(b) Bone model creation

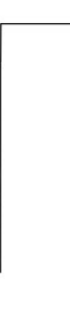

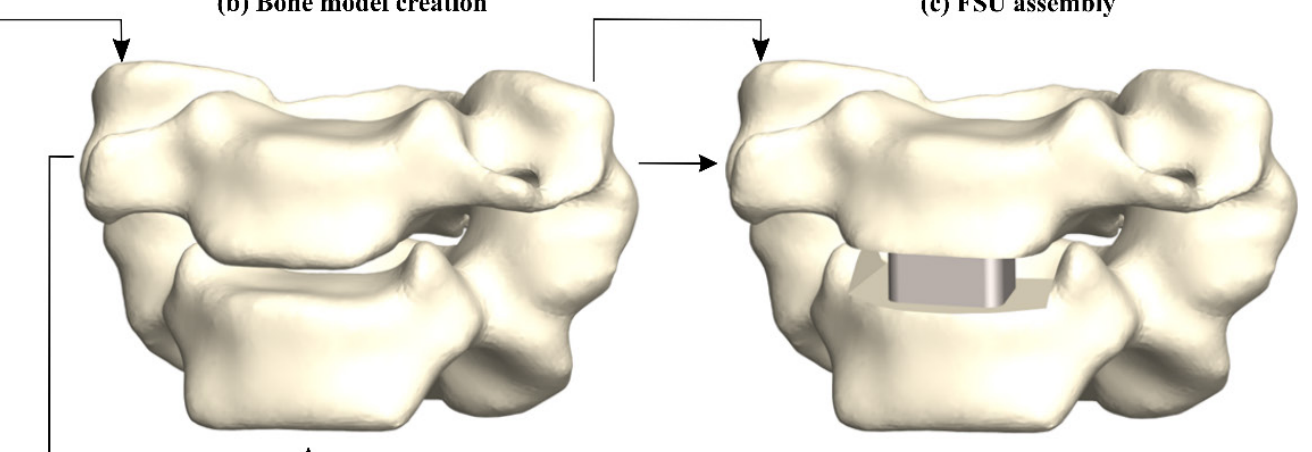

(c) FSU assembly
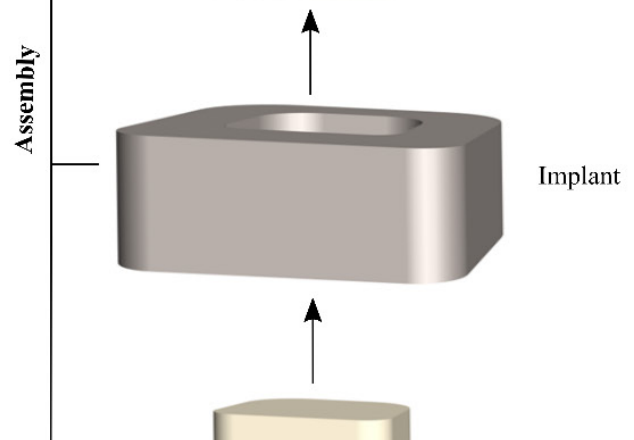

Bone graft

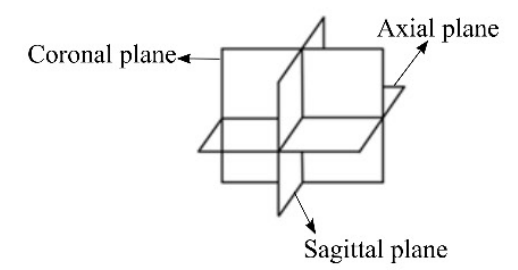

Figure B.1: Model creation and implant insertion. (a) Segmentation of C6-C7 vertebrae from axial CT-scan data; (b) 3D reconstructed vertebral model assembly with implant and bone graft; (c) Complete FSU after surgical insertion.

The approach of computing the $H U$ value of each element in the C6-C7 vertebral mesh was performed by arithmetically averaging the $H U$ of all nodes of the element. The $H U$ of each node is calculated by finding the nearest $\mathrm{CT}$ sampling point to the coordinates of each node, and assigning the value of the $\mathrm{CT}$ number $(H U)$ to the node. By manually investigating the current 
female CT dataset of the C6-C7 cervical vertebrae (Figure B.2), the distribution of $H U$ can be assumed as [67]:

$$
H U=\left\{\begin{array}{l}
\geq 1500, \quad \text { cortical bone } \\
<1500, \quad \text { cancellous bone }
\end{array}\right.
$$

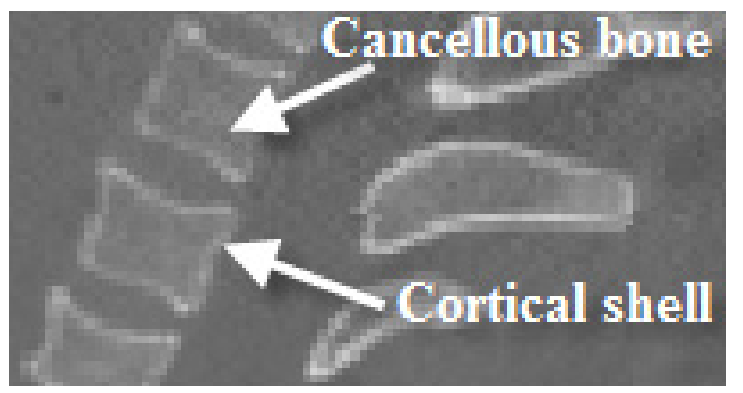

Figure B.2: Anatomical structure of C6-C7 vertebrae from the VHP of the US national library of medicine (NLM, Bethesda, Maryland, USA)

Each voxel in the CT-data has a grey/Hounsfield value that corresponds to BMD and accounts for its anatomical location [35]. Hounsfield unit values were converted to the corresponding bone mineral density using a linear regression between $H U$ and BMD [34]. The BMD of $0.499 \mathrm{~g} / \mathrm{cm}^{3}$ [71], for the female dataset, was assumed and assigned to the maximum $H U$ of 2448. BMD from quantitative computed tomography (QCT) in the $H U$ range (HU 852 to $H U 2448$ ) could be determined through the linear relationship (B.1) (Figure B.3):

$$
B M D_{Q C T}=\frac{H U}{2448} \times 0.499, \quad\left(\mathrm{~g} / \mathrm{cm}^{3}\right)
$$

Once the QCT mineral density values were calculated for all bone voxels, the following relation was used to obtain the axial elastic modulus $\left(E_{Z}, M P a\right)[34,44]$ :

$E_{Z}=-34.7+3230 B M D_{Q C T}$

Assuming transverse isotropy for bone, the other 4 elastic constants were calculated as [34, 42 , 43]:

$$
\begin{aligned}
& E_{X}=E_{Y}=0.333 E_{Z} \\
& v_{X Y}=0.381 \\
& v_{Y Z}=v_{X Z}=0.104 \\
& G_{X Y}=E_{X} / 2\left(1+v_{X Y}\right) \\
& G_{Y Z}=G_{X Z}=0.157 E_{Z}
\end{aligned}
$$

The materials of the facet joints was assumed isotropic with Young's modulus $E=5 \mathrm{MPa}$ and Poisson's ration $v=0.45$ [29]. Figure B.4 shows the material properties assigned to each element based on its $H U$ value, while the distribution of the mechanical properties over the vertebral bone is depicted in Figure B.5. 
(a)

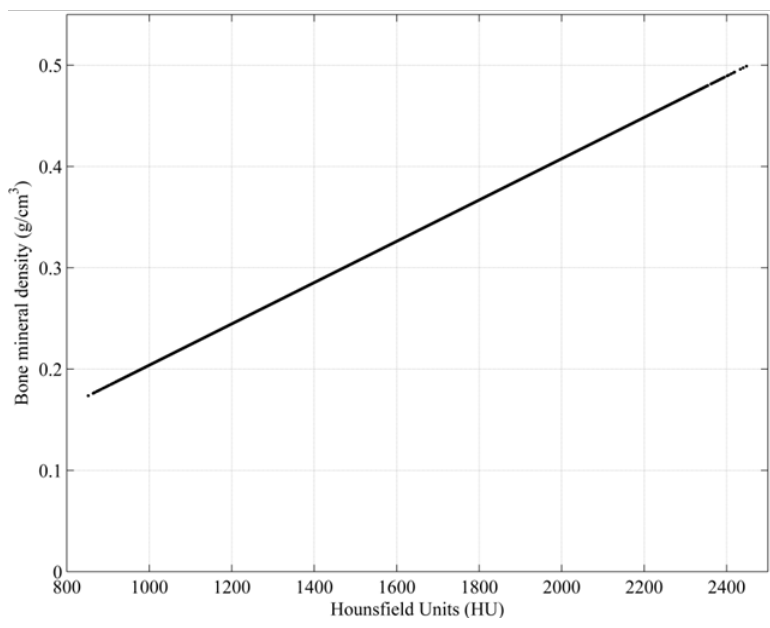

(b)

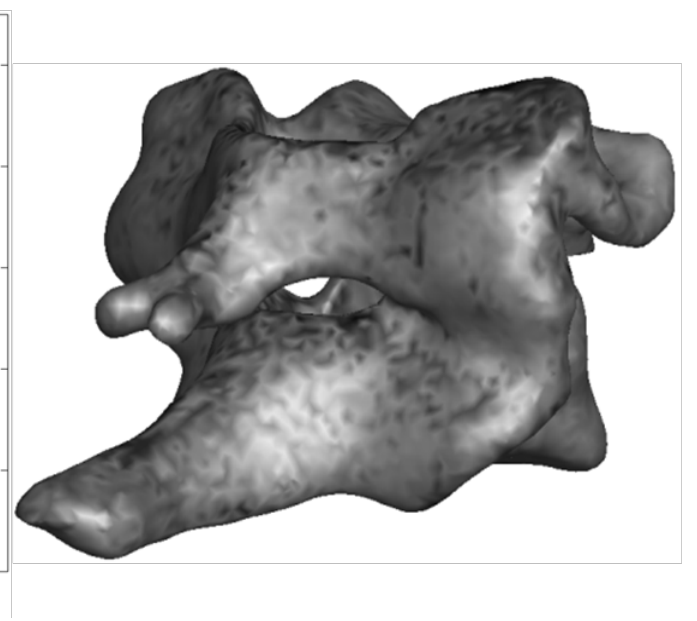

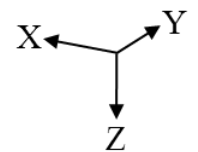

2400

2200

2000

1800

1600

1400

1200

1000

800

600

400

200

HU

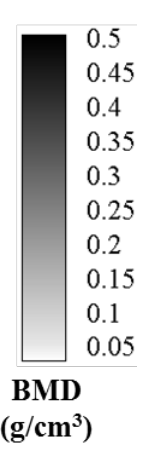

Figure B.3: (a) Linear interpolation between the Hounsfield unit (HU) values and BMD $\left(\mathrm{g} / \mathrm{cm}^{3}\right)$; (b) HU and BMD distributions over the C6-C7 vertebrae.

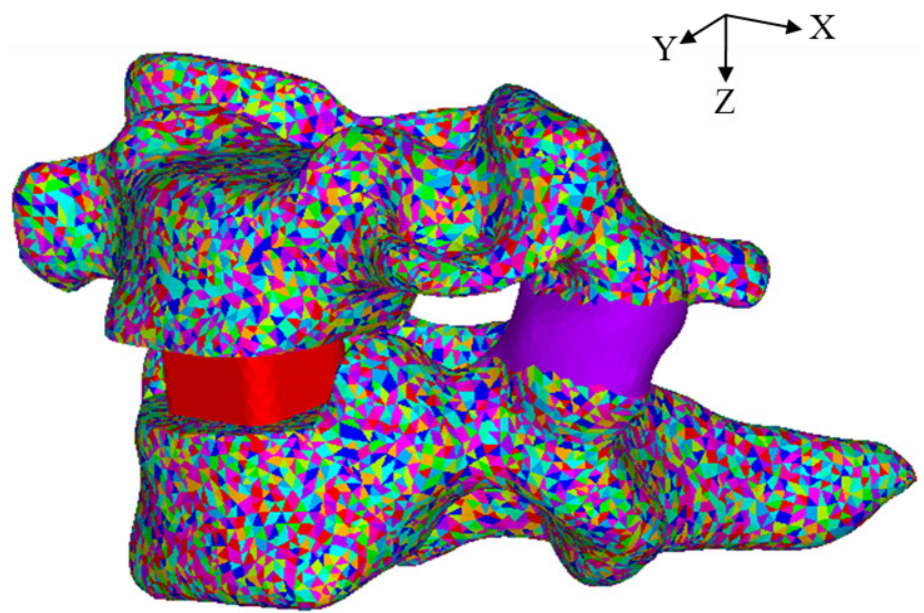

Figure B.4: Assigned material properties for each element of the cervical vertebrae 

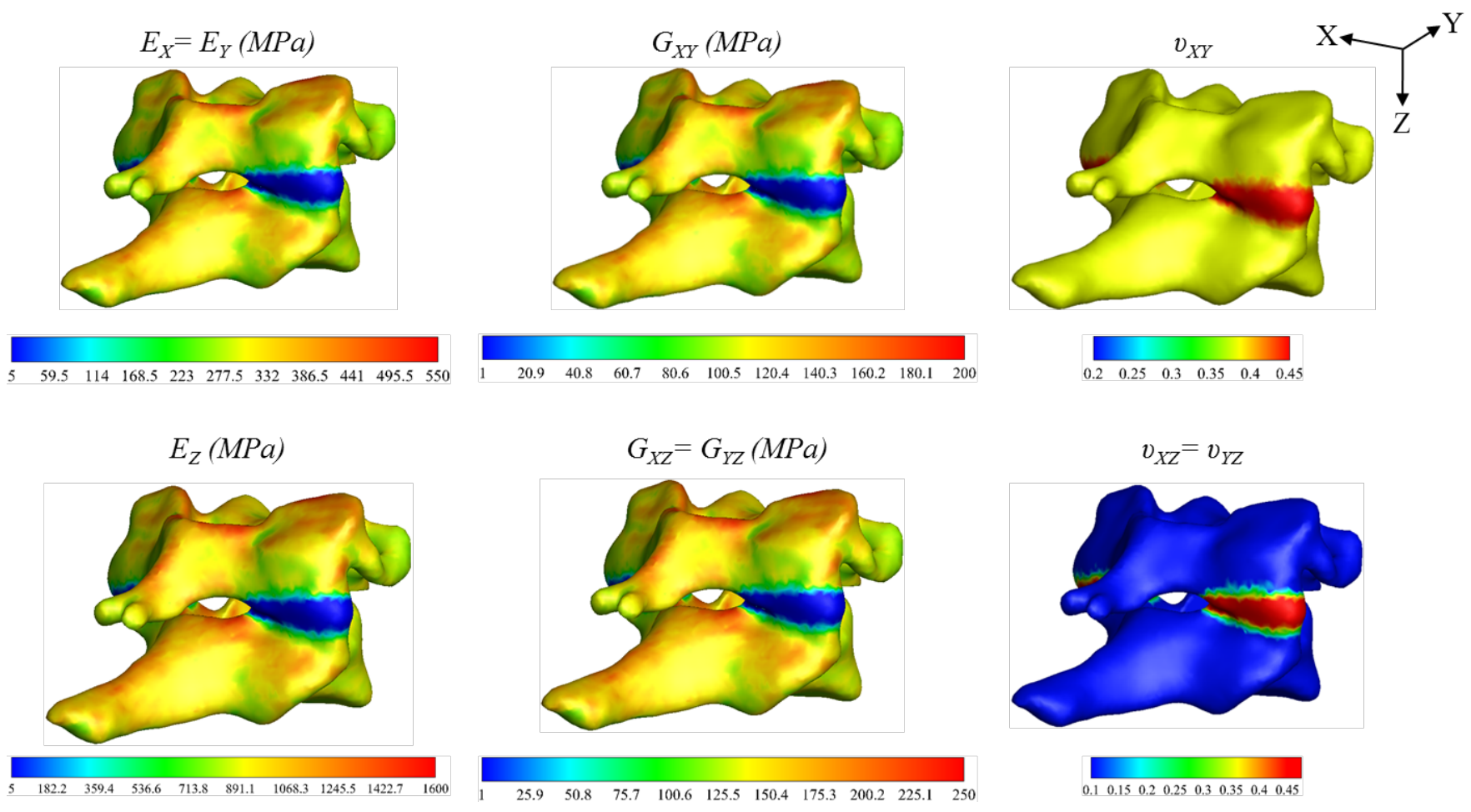

Figure B.5: Mechanical properties of the C6-C7 vertebrae.

\section{Appendix C: Objective function convergence}

The iteration history that shows the objective (compliance maximization) and its sensitivity along the optimization process is here reported for flexion, extension, right lateral bending, flexion combined with right lateral bending, and extension combined with right lateral bending load cases under the assigned compressive force (Figure C.1). The optimization loop is terminated if the change in the design variables $\rho_{e}(e=1 \ldots, N)$ is less than $1 \%$. Otherwise, the optimization steps are repeated. 
Flexion

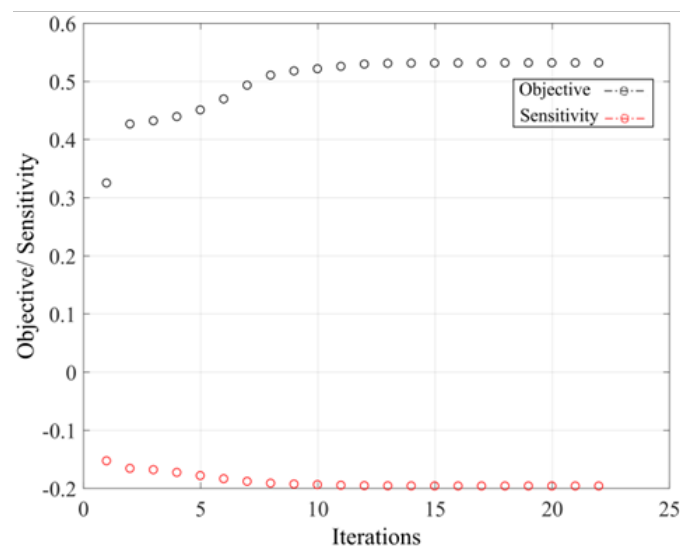

Flexion + Right lateral bending

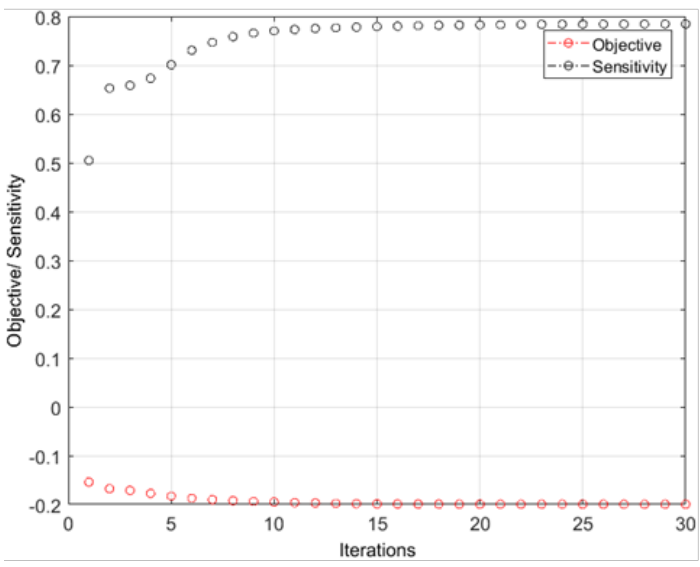

Extension

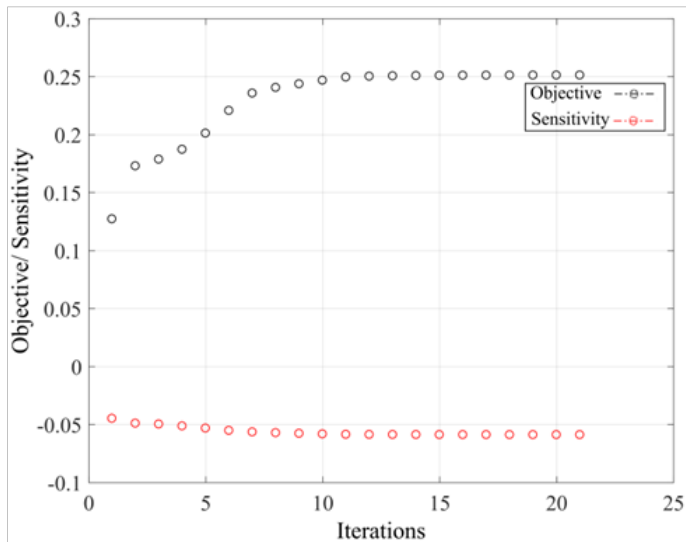

Lateral bending

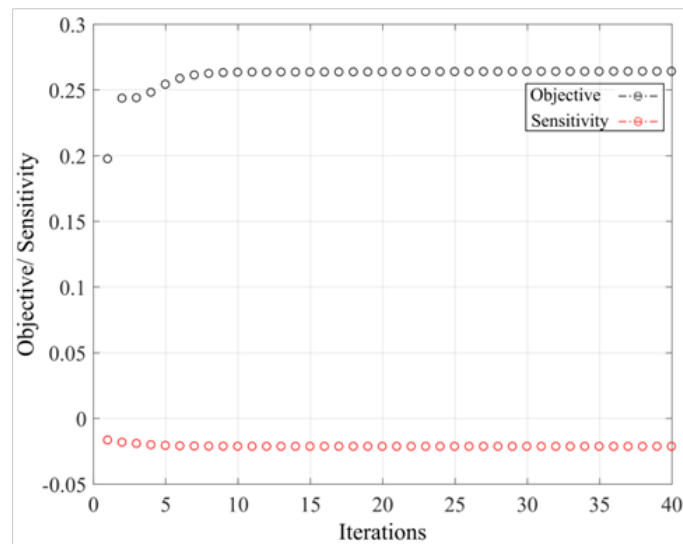

\section{Extension + Right lateral bending}

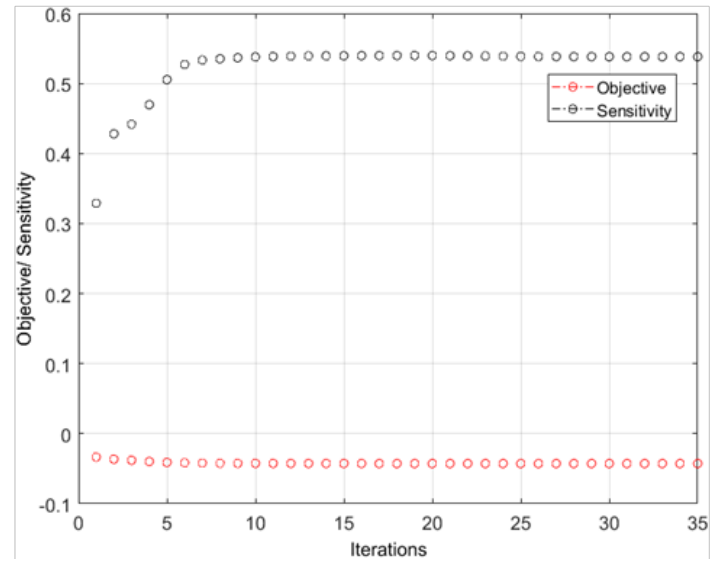

Figure C.1: Iteration history for the objective (compliance maximization) and sensitivity, for flexion, extension, right lateral bending, flexion with right lateral bending, and extension with right lateral bending modes. 


\section{Appendix D: Effective yield properties and failure analysis of implant microstructure}

\section{Effective yield properties}

Similarly to the analysis of the elastic properties provided in section 2.2 , the microscopic stress tensor can be obtained via $\mathrm{AH}$ and written as:

$\sigma_{i j}=E_{i j k l} M_{k l m n} \bar{\varepsilon}_{m n}$

Using the above, and replacing $\bar{\varepsilon}_{k l}$ with the effective stress tensor $\bar{\sigma}_{i j}$ and the homogenized elastic tensor, $E_{i j k l}^{H}$, in (3) yields the following simplified relationship between the microscopic stress distribution and the macroscopic stress tensor:

$\sigma_{i j}=E_{i j k l} M_{k l m n}\left(E_{r s m n}^{H}\right)^{-1} \bar{\sigma}_{r s}$

wherein the $\bar{\sigma}_{r s}$ is the macroscopic stress distribution through the unit cell of the lattice domain. If $\sigma_{y s}$ is the yield strength of the cell walls, the yield surface of the unit cell can be written from (D.2) as:

$\bar{\sigma}_{i j}^{y}=\frac{\sigma_{y s}}{\max \left\{\sigma_{\mathrm{vM}}\left(\bar{\sigma}_{i j}\right)\right\}} \bar{\sigma}_{i j}$

where the $\sigma_{\mathrm{vM}}\left(\bar{\sigma}_{i j}\right)$ is the von-Mises stress distribution within the unit cell corresponding to the macroscopic stress $\bar{\sigma}_{i j}$. Figure D.1 illustrates the predicted yield strength of the tetrahedron based cell topology used in this work [57], all normalized with the yield strength, $\sigma_{y s}$, of the solid material, and expressed as a function of relative density $\rho \cdot \bar{\sigma}_{x x}^{y}, \bar{\sigma}_{y y}^{y}, \bar{\sigma}_{z z}^{y}$ are the yield strengths in the $\mathrm{x}, \mathrm{y}, \mathrm{z}$ direction, $\bar{\sigma}_{x y}^{y}, \bar{\sigma}_{y z}^{y}, \bar{\sigma}_{x z}^{y}$ are the shear strengths in the three planes of symmetry, which have identical magnitude, and $\bar{\sigma}_{b x y}^{y}, \bar{\sigma}_{b y z}^{y}, \bar{\sigma}_{b x z}^{y}$ are the shear strengths in the bi-axial directions. 


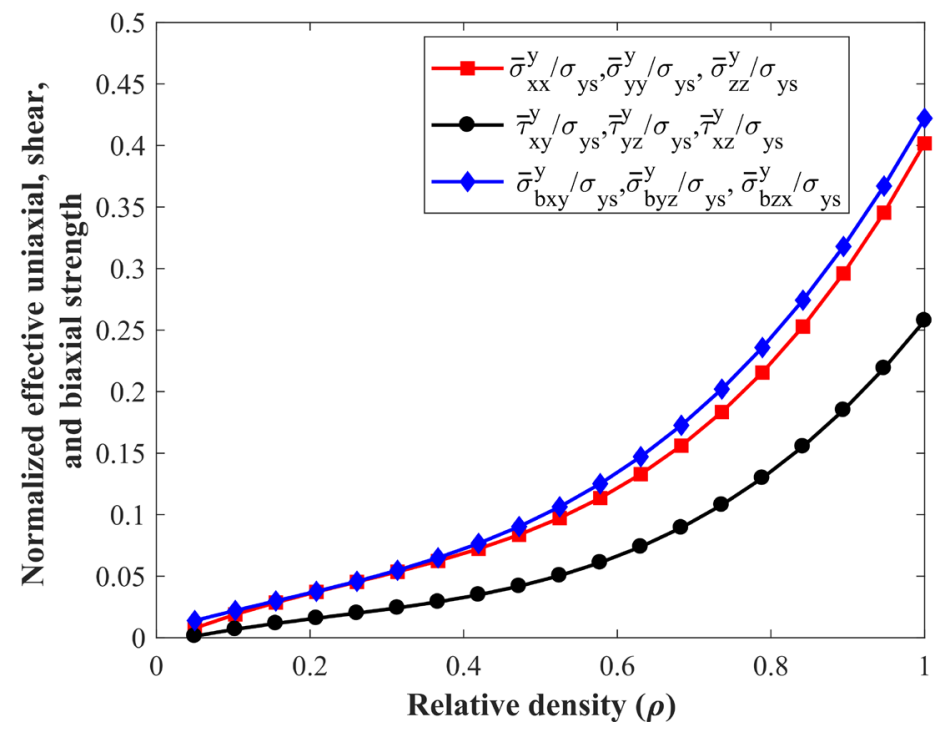

Figure D.1: Normalized effective yield strength of the tetrahedron based unit cell as a function of relative density.

\section{Tsai-Wu Failure criterion}

The yield strength of a tetrahedron lattice under multiaxial loading conditions can be obtained by solving the local problem defined in Equation 5. Given the time required to solve this problem for each loading direction, in this work we resort to the Tsai-Wu failure criterion, a metric of failure widely used for anisotropic materials and here applied to the tetrahedron lattice under multiaxial conditions [57]. Because the tetrahedron based cell of the lattice cage has three planes of symmetry, the general form of the Tsai-Wu failure criterion reduces to [72]:

$F_{i} \bar{\sigma}_{i}+F_{i j} \bar{\sigma}_{i} \bar{\sigma}_{j} \leq 1 / S F$

where $i, j=1 \ldots 6$ are repeated indices to indicate summation, and $F_{i}, F_{i j}$ are experimentally determined material strength parameters. The stresses $\bar{\sigma}_{i}$ are expressed in Voigt notation. For orthotropic materials with three planes of symmetry, if we assume that $F_{i j}=F_{j i}$ and no coupling exists between the normal and shear stress terms, the Tsai-Wu failure criterion can be written as:

$F_{1} \bar{\sigma}_{1}+F_{2} \bar{\sigma}_{2}+F_{3} \bar{\sigma}_{3}+F_{4} \bar{\sigma}_{4}+F_{5} \bar{\sigma}_{5}+F_{6} \bar{\sigma}_{6}+$
$F_{11} \bar{\sigma}_{1}^{2}+F_{22} \bar{\sigma}_{2}^{2}+F_{33} \bar{\sigma}_{3}^{2}+F_{44} \bar{\sigma}_{4}^{2}+F_{55} \bar{\sigma}_{5}^{2}+F_{66} \bar{\sigma}_{6}^{2}+$
$2 F_{12} \bar{\sigma}_{1} \bar{\sigma}_{2}+2 F_{13} \bar{\sigma}_{1} \bar{\sigma}_{3}+2 F_{23} \bar{\sigma}_{2} \bar{\sigma}_{3} \leq 1 / S F$ 
We assume the yield strengths of the unit cell in tension and compression are equal, and the yield strength in the $\mathrm{x}, \mathrm{y}, \mathrm{z}$ directions can be noted as $\bar{\sigma}_{x x}^{y}, \bar{\sigma}_{y y}^{y}, \bar{\sigma}_{z z}^{y}$. We also assume the shear strengths in the three planes of symmetry have identical magnitude even if the signs are different and are noted as $\bar{\sigma}_{x y}^{y}, \bar{\sigma}_{y z}^{y}, \bar{\sigma}_{x z}^{y}$. The coefficients of the Tsai-Wu failure criterion are then given by:

$$
\begin{aligned}
& F_{1}=F_{2}=F_{3}=F_{4}=F_{5}=F_{6}=0 \\
& F_{11}=\frac{1}{\left(\bar{\sigma}_{x x}^{y}\right)^{2}}, F_{22}=\frac{1}{\left(\bar{\sigma}_{y y}^{y}\right)^{2}}, F_{33}=\frac{1}{\left(\bar{\sigma}_{z z}^{y}\right)^{2}} \\
& F_{44}=\frac{1}{\left(\bar{\sigma}_{x y}^{y}\right)^{2}}, F_{55}=\frac{1}{\left(\bar{\sigma}_{y z}^{y}\right)^{2}}, F_{66}=\frac{1}{\left(\bar{\sigma}_{x z}^{y}\right)^{2}}
\end{aligned}
$$

The coefficients $F_{12}, F_{23}, F_{13}$ can be determined via equibiaxial tests. If the failure strengths in equibiaxial tension are $\bar{\sigma}_{1}=\bar{\sigma}_{2}=\bar{\sigma}_{b x y}^{y}, \bar{\sigma}_{1}=\bar{\sigma}_{3}=\bar{\sigma}_{b x z}^{y}, \bar{\sigma}_{2}=\bar{\sigma}_{3}=\bar{\sigma}_{b y z}^{y}$ then:

$$
\begin{aligned}
& F_{12}=\frac{1}{2\left(\bar{\sigma}_{b x y}^{y}\right)^{2}}\left[1-\left(\bar{\sigma}_{b x y}^{y}\right)^{2}\left(F_{11}+F_{22}\right)\right] \\
& F_{23}=\frac{1}{2\left(\bar{\sigma}_{b y z}^{y}\right)^{2}}\left[1-\left(\bar{\sigma}_{b y z}^{y}\right)^{2}\left(F_{22}+F_{33}\right)\right] \\
& F_{13}=\frac{1}{2\left(\bar{\sigma}_{b x z}^{y}\right)^{2}}\left[1-\left(\bar{\sigma}_{b x z}^{y}\right)^{2}\left(F_{11}+F_{33}\right)\right]
\end{aligned}
$$

The above procedure enables the verification of the safety facture for the fusion cage concepts here examined under the various loading conditions. The minimum safety factor is found to be 3.25 in the case of flexion combined with the right lateral bending moment load case. 


\section{References}

1. Chong, E., M.H. Pelletier, R.J. Mobbs, and W.R. Walsh, The design evolution of interbody cages in anterior cervical discectomy and fusion: a systematic review. BMC Musculoskeletal Disorders, 2015. 16(1): p. 99.

2. Irvine, D.H., J.B. Foster, D.J. Newell, and B.N. Klukvin, Prevalence of cervical spondylosis in a general practice. The Lancet, 1995. 285(7395): p. 1089-1092.

3. Chau, A.M.T. and R.J. Mobbs, Bone graft substitutes in anterior cervical discectomy and fusion. European Spine Journal, 2009. 18(4): p. 449-464.

4. Fountas, K.N., E.Z. Kapsalaki, L.G. Nikolakakos, H.F. Smisson, K.W. Johnston, A.A. Grigorian, G.P. Lee, and J.S. Robinson, Jr., Anterior cervical discectomy and fusion associated complications. Spine (Phila Pa 1976), 2007. 32(21): p. 2310-7.

5. Bertagnoli, R., J.J. Yue, R.V. Shah, R. Nanieva, F. Pfeiffer, A. Fenk-Mayer, T. Kershaw, and D.S. Husted, The treatment of disabling single-level lumbar discogenic low back pain with total disc arthroplasty utilizing the Prodisc prosthesis: a prospective study with 2-year minimum follow-up. Spine (Phila Pa 1976), 2005. 30(19): p. 2230-6.

6. Smith, G.W. and R.A. Robinson, The treatment of certain cervical-spine disorders by anterior removal of the intervertebral disc and interbody fusion. J Bone Joint Surg Am, 1958. 40-a(3): p. 607-24.

7. Chong, E., R.J. Mobbs, M.H. Pelletier, and W.R. Walsh, Titanium/Polyetheretherketone Cages for Cervical Arthrodesis with Degenerative and Traumatic Pathologies: Early Clinical Outcomes and Fusion Rates. Orthopaedic Surgery, 2016. 8(1): p. 19-26.

8. Anderson, P.A. and J.P. Rouleau, Intervertebral Disc Arthroplasty. Spine, 2004. 29(23): p. 2779-2786.

9. Whitecloud, T.S., 3rd, J.M. Davis, and P.M. Olive, Operative treatment of the degenerated segment adjacent to a lumbar fusion. Spine (Phila Pa 1976), 1994. 19(5): $\mathrm{p}$. 531-6.

10. McConnell, J.R., B.J. Freeman, U.K. Debnath, M.P. Grevitt, H.G. Prince, and J.K. Webb, A prospective randomized comparison of coralline hydroxyapatite with autograft in cervical interbody fusion. Spine (Phila Pa 1976), 2003. 28(4): p. 317-23.

11. Bartels, R.H., R.D. Donk, and T. Feuth, Subsidence of stand-alone cervical carbon fiber cages. Neurosurgery, 2006. 58.

12. Ryu, S.I., M. Mitchell, and D.H. Kim, A prospective randomized study comparing a cervical carbon fiber cage to the Smith-Robinson technique with allograft and plating: up to 24 months follow-up. European Spine Journal, 2006. 15(2): p. 157-164.

13. Gercek, E., V. Arlet, J. Delisle, and D. Marchesi, Subsidence of stand-alone cervical cages in anterior interbody fusion: warning. Eur Spine J, 2003. 12.

14. Parsons, J.R., S. Bhayani, H. Alexander, and A.B. Weiss, Carbon fiber debris within the synovial joint. A time-dependent mechanical and histologic study. Clin Orthop Relat Res, 1985(196): p. 69-76.

15. Rao, P.J., M.H. Pelletier, W.R. Walsh, and R.J. Mobbs, Spine interbody implants: material selection and modification, functionalization and bioactivation of surfaces to improve osseointegration. Orthop Surg, 2014. 6(2): p. 81-9.

16. Svehla, M., P. Morberg, B. Zicat, W. Bruce, D. Sonnabend, and W.R. Walsh, Morphometric and mechanical evaluation of titanium implant integration: comparison of five surface structures. J Biomed Mater Res, 2000. 51(1): p. 15-22. 
17. Kurtz, S.M. and J.N. Devine, PEEK Biomaterials in Trauma, Orthopedic, and Spinal Implants. Biomaterials, 2007. 28(32): p. 4845-4869.

18. Park, J.-I., D.-C. Cho, K.-T. Kim, and J.-K. Sung, Anterior Cervical Discectomy and Fusion Using a Stand-Alone Polyetheretherketone Cage Packed with Local Autobone : Assessment of Bone Fusion and Subsidence. Journal of Korean Neurosurgical Society, 2013. 54(3): p. 189-193.

19. Chou, Y.C., D.C. Chen, W.A. Hsieh, W.F. Chen, P.S. Yen, T. Harnod, T.L. Chiou, Y.L. Chang, C.F. Su, S.Z. Lin, and S.Y. Chen, Efficacy of anterior cervical fusion: comparison of titanium cages, polyetheretherketone (PEEK) cages and autogenous bone grafts. J Clin Neurosci, 2008. 15(11): p. 1240-5.

20. Niu, C.C., J.C. Liao, W.J. Chen, and L.H. Chen, Outcomes of interbody fusion cages used in 1 and 2-levels anterior cervical discectomy and fusion: titanium cages versus polyetheretherketone (PEEK) cages. J Spinal Disord Tech, 2010. 23(5): p. 310-6.

21. Liao, J.-C., C.-C. Niu, W.-J. Chen, and L.-H. Chen, Polyetheretherketone (PEEK) cage filled with cancellous allograft in anterior cervical discectomy and fusion. International Orthopaedics, 2008. 32(5): p. 643-648.

22. Cabraja, M., S. Oezdemir, D. Koeppen, and S. Kroppenstedt, Anterior cervical discectomy and fusion: comparison of titanium and polyetheretherketone cages. BMC Musculoskelet Disord, 2012. 13: p. 172.

23. Kotsias, A., S. Mularski, B. Kühn, M. Hanna, and O. Suess, Does partial coating with titanium improve the radiographic fusion rate of empty PEEK cages in cervical spine surgery? A comparative analysis of clinical data. Patient Safety in Surgery, 2017. 11(1): p. 13.

24. Wilke, H.J., A. Kettler, and L. Claes, Primary stabilizing effect of interbody fusion devices for the cervical spine: an in vitro comparison between three different cage types and bone cement. Eur Spine J, 2000. 9(5): p. 410-6.

25. Wilke HJ, K.A., Goetz C, Claes L., Subsidence Resulting From Simulated Postoperative Neck Movements: An In Vitro Investigation With a New Cervical Fusion Cage. 2000. 25(21): p. 2762-2770.

26. Kettler, A., H.J. Wilke, and L. Claes, Effects of neck movements on stability and subsidence in cervical interbody fusion: an in vitro study. J Neurosurg, 2001. 94(1 Suppl): p. 97-107.

27. Furderer, S., F. Schollhuber, J.D. Rompe, and P. Eysel, Effect of design and implantation technique on risk of progressive sintering of various cervical vertebrae cages. Orthopade, 2002. 31(5): p. 466-71.

28. Lin, C.Y., C.C. Hsiao, P.Q. Chen, and S.J. Hollister, Interbody fusion cage design using integrated global layout and local microstructure topology optimization. Spine (Phila Pa 1976), 2004. 29(16): p. 1747-54.

29. Lin, C.-Y., H. Kang, J.P. Rouleau, S.J. Hollister, and F.L. Marca, Stress Analysis of the Interface Between Cervical Vertebrae End Plates and the Bryan, Prestige LP, and ProDisc-C Cervical Disc Prostheses: An In Vivo Image-Based Finite Element Study. Spine, 2009. 34(15): p. 1554-1560.

30. Chiang, M.-F., J.-M. Teng, C.-H. Huang, C.-K. Cheng, C.-S. Chen, T.-K. Chang, and S.H. Chao, Finite Element Analysis of Cage Subsidence in Cervical Interbody Fusion. Journal of Medical and Biological Engineering, 2004. 24(2): p. 201-208. 
31. Arabnejad, S., R. Burnett Johnston, J.A. Pura, B. Singh, M. Tanzer, and D. Pasini, Highstrength porous biomaterials for bone replacement: A strategy to assess the interplay between cell morphology, mechanical properties, bone ingrowth and manufacturing constraints. Acta Biomater, 2016. 30: p. 345-356.

32. Hassani, B.H., E., a review of homogenization and topology optimization Ihomogenization theory for media with periodic structure. 1997.

33. Hollister, S.J. and N. Kikuchi, A comparison of homogenization and standard mechanics analyses for periodic porous composites. Computational Mechanics, 1992. 10(2): p. 7395.

34. Crawford, R.P., C.E. Cann, and T.M. Keaveny, Finite element models predict in vitro vertebral body compressive strength better than quantitative computed tomography. Bone, 2003. 33(4): p. 744-50.

35. Rho, J.Y., M.C. Hobatho, and R.B. Ashman, Relations of mechanical properties to density and CT numbers in human bone. Med Eng Phys, 1995. 17(5): p. 347-355.

36. Arabnejad Khanoki, S. and D. Pasini, Fatigue design of a mechanically biocompatible lattice for a proof-of-concept femoral stem. J Mech Behav Biomed Mater, 2013. 22: p. 65-83.

37. Arabnejad, S. and D. Pasini, Mechanical properties of lattice materials via asymptotic homogenization and comparison with alternative homogenization methods. International Journal of Mechanical Sciences, 2013. 77: p. 249-262.

38. Bendsøe, M.P., Optimization of Structural Topology, Shape, and Material. 1995: Springer.

39. Paul A. Yushkevich, Joseph Piven, Heather Cody Hazlett, Rachel Gimpel Smith, Sean Ho, James C. Gee, and a.G. Gerig, User-guided 3D active contour segmentation of anatomical structures: Significantly improved efficiency and reliability. Neuroimage, 2006. 31(3): p. 1116-28.

40. P. Cignoni, M.C., M. Corsini, M. Dellepiane, F. Ganovelli, G. Ranzuglia, MeshLab: an Open-Source Mesh Processing Tool. Sixth Eurographics Italian Chapter Conference, 2008: p. 129-136.

41. Yang and King-Hay, Basic finite element method as applied to injury biomechanics. 2017.

42. Mosekilde, L. and C.C. Danielsen, Biomechanical competence of vertebral trabecular bone in relation to ash density and age in normal individuals. Bone, 1987. 8(2): p. 79-85.

43. Ulrich, D., B. van Rietbergen, A. Laib, and P. Ruegsegger, The ability of threedimensional structural indices to reflect mechanical aspects of trabecular bone. Bone, 1999. 25(1): p. 55-60.

44. Kopperdahl, D.L., E.F. Morgan, and T.M. Keaveny, Quantitative computed tomography estimates of the mechanical properties of human vertebral trabecular bone. J Orthop Res, 2002. 20(4): p. 801-5.

45. Moroney, S.P., A.B. Schultz, J.A.A. Miller, and G.B.J. Andersson, Load-displacement properties of lower cervical spine motion segments. J Biomech, 1988. 21(9): p. 769-779.

46. Guedes, J. and N. Kikuchi, Preprocessing and postprocessing for materials based on the homogenization method with adaptive finite element methods. Computer methods in applied mechanics and engineering, 1990. 83(2): p. 143-198. 
47. Hassani, B., A direct method to derive the boundary conditions of the homogenization equation for symmetric cells. Communications in numerical methods in engineering, 1996. 12(3): p. 185-196.

48. Arabnejad Khanoki, S. and D. Pasini, Multiscale Design and Multiobjective Optimization of Orthopedic Hip Implants with Functionally Graded Cellular Material. J Biomech Eng, 2012. 134(3): p. 031004-031004-10.

49. Arabnejad, S., B. Johnston, M. Tanzer, and D. Pasini, Fully porous 3D printed titanium femoral stem to reduce stress-shielding following total hip arthroplasty. Journal of Orthopaedic Research, 2016. 35(8): p. 1774-1783.

50. Deshpande, V.S., N.A. Fleck, and M.F. Ashby, Effective properties of the octet-truss lattice material. Journal of the Mechanics and Physics of Solids, 2001. 49(8): p. 17471769.

51. Bendsøe, M.P. and O. Sigmund, Topology optimization : theory, methods, and applications. 2003, Berlin; New York: Springer.

52. Sigmund, O. and J. Petersson, Numerical instabilities in topology optimization: A survey on procedures dealing with checkerboards, mesh-dependencies and local minima. Structural optimization, 1998. 16(1): p. 68-75.

53. Sigmund, O., Morphology-based black and white filters for topology optimization. Structural and Multidisciplinary Optimization, 2007. 33(4-5): p. 401-424.

54. Sigmund, O., Design of material structures using topology optimization, in Department of Solid Mechanics. 1994, Technical University of Denmark: Lyngby, Denmark.

55. Sigmund, O., On the Design of Compliant Mechanisms Using Topology Optimization. Mechanics of Structures and Machines, 1997. 25(4): p. 493-524.

56. Melancon, D., Z. Bagheri, R. Johnston, L. Liu, M. Tanzer, and D. Pasini, Mechanical characterization of structurally porous biomaterials built via additive manufacturing: experiments, predictive models, and design maps for load-bearing bone replacement implants. Acta Biomater, 2017.

57. Arabnejad, S., B. Johnston, M. Tanzer, and D. Pasini, Fully porous 3D printed titanium femoral stem to reduce stress-shielding following total hip arthroplasty. J Orthop Res, 2017. 35(8): p. 1774-1783.

58. Goel, V.K. and J.D. Clausen, Prediction of load sharing among spinal components of a C5-C6 motion segment using the finite element approach. Spine (Phila Pa 1976), 1998. 23(6): p. 684-91.

59. Naoki, T., Z. Masaru, and O. Yoshihiro, Multi-scale finite element analysis of porous materials and components by asymptotic homogenization theory and enhanced mesh superposition method. Modelling and Simulation in Materials Science and Engineering, 2003. 11(2): p. 137.

60. Sobral, J.M., S.G. Caridade, R.A. Sousa, J.F. Mano, and R.L. Reis, Three-dimensional plotted scaffolds with controlled pore size gradients: Effect of scaffold geometry on mechanical performance and cell seeding efficiency. Acta Biomater, 2011. 7(3): p. 10091018.

61. Khoda, A.K., I.T. Ozbolat, and B. Koc, Engineered tissue scaffolds with variational porous architecture. J Biomech Eng, 2011. 133(1): p. 011001.

62. Heinl, P., L. Müller, C. Körner, R.F. Singer, and F.A. Müller, Cellular Ti-6Al-4V structures with interconnected macro porosity for bone implants fabricated by selective electron beam melting. Acta Biomater, 2008. 4(5): p. 1536-1544. 
63. de Wild, M., R. Schumacher, K. Mayer, E. Schkommodau, D. Thoma, M. Bredell, A. Kruse Gujer, K.W. Gratz, and F.E. Weber, Bone regeneration by the osteoconductivity of porous titanium implants manufactured by selective laser melting: a histological and micro computed tomography study in the rabbit. Tissue Eng Part A, 2013. 19(23-24): p. 2645-54.

64. Murr, L.E., S.M. Gaytan, F. Medina, H. Lopez, E. Martinez, B.I. Machado, D.H. Hernandez, L. Martinez, M.I. Lopez, R.B. Wicker, and J. Bracke, Next-generation biomedical implants using additive manufacturing of complex, cellular and functional mesh arrays. Philosophical Transactions of the Royal Society A: Mathematical,

Physical and Engineering Sciences, 2010. 368(1917): p. 1999-2032.

65. Parthasarathy, J., B. Starly, S. Raman, and A. Christensen, Mechanical evaluation of porous titanium (Ti6Al4V) structures with electron beam melting (EBM). J Mech Behav Biomed Mater, 2010. 3(3): p. 249-259.

66. Hughes, T.J.R., The Finite Element Method: Linear Static and Dynamic Finite Element Analysis: Thomas J. R. Hughes. Computer-Aided Civil and Infrastructure Engineering, 1989. 4(3): p. 245-246.

67. Peng, L., J. Bai, X. Zeng, and Y. Zhou, Comparison of isotropic and orthotropic material property assignments on femoral finite element models under two loading conditions. Med Eng Phys, 2006. 28(3): p. 227-233.

68. Viceconti, M., L. Bellingeri, L. Cristofolini, and A. Toni, A comparative study on different methods of automatic mesh generation of human femurs. Med Eng Phys, 1998. 20(1): p. 1-10.

69. Odgaard, A., Three-dimensional methods for quantification of cancellous bone architecture. Bone, 1997. 20(4): p. 315-328.

70. Lengsfeld, M., J. Schmitt, P. Alter, J. Kaminsky, and R. Leppek, Comparison of geometry-based and CT voxel-based finite element modelling and experimental validation. Med Eng Phys, 1998. 20(7): p. 515-522.

71. Anderst, W.J., E. Thorhauer, J. Lee, W. Donaldson, and J. Kang, Cervical Spine Bone Mineral Density as a Function of Vertebral Level and Anatomic Location. The spine journal : official journal of the North American Spine Society, 2011. 11(7): p. 659-667.

72. Tsai, S.W. and E.M. Wu, A general theory of strength for anisotropic materials. Journal of composite materials, 1971. 5(1): p. 58-80. 